\title{
RECENT DEVELOPMENTS IN SOME NON-SELF-ADJOINT PROBLEMS OF MATHEMATICAL PHYSICS
}

\author{
C. L. DOLPH
}

1. Introduction. In view of the fact that a relatively small percentage of the membership of the American Mathematical Society have an opportunity to become acquainted with or follow the ever increasing activity in the fields of applied and immediately applicable mathematics, it is perhaps useful to attempt an assessment of recent trends in the areas with which I have some familiarity, namely in the field of diffraction and scattering theory and that part of transport theory where similar techniques are employed. While one would need the prescience of a Hilbert to adequately carry out even this limited program, it is possible to pick out a few concepts and techniques which underlie many of the recent developments.

The areas under consideration received a tremendous impetus as a result of the radar development of the last war and more recently as a result of reactor theory and the attempts to achieve controlled fusion. In many instances, it was the physicists and not the mathematicians who led the way to new methods which in some cases are only formally understood mathematically to this day even though they may have been widely applied with frequent success. This area is also characterized by the fact that the tools necessary to establish recent results have frequently been available for many years. This does not detract from the achievement of the authors who found this kind of result but once again illustrates the healthy influence physical problems can have on the development of mathematics and the dangers inherent when the two get too widely divorced. Finally, there are instances where mathematicians have labored arduously to establish a rigorous theory only to find a physicist with more insight into the problem rendering their work unnecessary by being less tradition minded and redoing the problem in a more appropriate space.

By way of illustration of the first point of the last paragraph, one has the work of Booker [4] and Furry [IX] on normal mode propagation through an inhomogeneous atmosphere, the work of Peierls and Kapur [82], Siegert [91] and Wigner [107] on compound nuclei, the work of Schwinger on variational principles [65], the formalism of scattering theory by Gell-Mann and Goldberger [32], after the

An address delivered before the Chicago meeting of the Society on April 23, 1960 by invitation of the Committee to Select Hour Speakers for Western Sectional Meetings; received by the editors May 30, 1960. 
introduction of the $S$-matrix concept by Heisenberg [38], the work of MacFarlane [71] on the Rayleigh-Ritz principle in normal mode theory, the work of Kohn $[48 ; 49]$ on variational principles, the work of Heitler [VI] in complex atomic reactions, the work of Weisskopf, Feshbach, and Porter [100] on the optical model of the nucleus, the work of Wightman [104], Bogoliubov [III], Khuri [47], and Mandelstam [72] on dispersion relations and the analytic continuation of functions of several complex variables, the work of Van Kampen $[98 ; 99]$ and Case $[10 ; 11]$ on transport phenomena to name only a few of the inputs from physicists.

To illustrate the second point, is it not surprising that after the beautiful theory developed by Fredholm circa 1900 for the existence and uniqueness of the various potential problems by the integral equation method, it was not until 1952 that Weyl [102] first proved and then Müller ${ }^{1}$ [76] simplified the corresponding results by the same method for the exterior problems of the scalar wave equation. The elegant results of Sims [92] on the Sturm-Liouville problem with complex $q(x)$ and complex boundary conditions is another instance in that the basic limit point, limit circle geometry of Weyl's [101] 1910 treatment is readily carried over to this new situation. As a final illustration consider the Plemelj formula, [85] discovered in 1908 as a result of Hilbert's work on his transforms. It was not until the appearance of Muskhelishvili's book [XII] in English in 1953 that the beautiful theory of singular integral equations was widely known here and to the best of my knowledge, we do not often teach these formulas even in intermediate complex variable theory to this day, although as we shall see they underlie much of the modern developments in the fields under discussion. This in spite of the fact that the basic ideas are already contained in the monographs by Carleman [9] dating from 1922.

Our first example will illustrate the disadvantages of a traditionally minded approach to a simple problem. A second example is furnished by the recent work of Case on hydrodynamical problems of stability. By abandoning the classical steady state approach and treating the initial value problem directly by means of Laplace transforms, Case was able to obtain distribution solutions in the inviscid limit which were limits of viscous solutions and thus justify the classical normalmode approach with the aid of the continuous spectra associated with his new class of solutions. A final example and one that has influenced

${ }^{1}$ Somewhat more accurately, Weyl's work of 1952 was the first known to the Western world although Kupradse seems to have developed the theory in 1943. See $[56]$ and $[\mathbf{X}]$. 
all subsequent developments in scattering theory to this day is the $S$-matrix introduced by Heisenberg [38] in 1943. As Friedrichs ${ }^{2}$ has remarked, it is incredible that this natural notion did not appear in the mathematical literature prior to this time.

Most of the post-war developments in the area in question have much in common. In the first place they are singular in the sense that they involve noncompact regions of one, two or three dimensional space although they may involve compact regions as well. More explicitly their solution involves consideration of exterior problems and the asymptotic behavior at infinity in contrast to mathematical physics prior to quantum theory which concentrated on interior problems for which a large mathematical discipline had been developed in such areas as potential theory, integral equation theory where a completely continuous iterated operator existed, and related fields. The new developments are also closely related to what has come to be called dissipative operators; namely operators whose associated hermitian quadratic form has the property that its imaginary part is at least semi-definite.

This class of operators arises naturally from the consideration of the free space Green function for the scalar wave equation which is fundamental to acoustic, electro-magnetic and quantum scattering theory and the dissipative property is a direct consequence of the radiation condition of Sommerfeld. (It is interesting to note that the use of the dissipative concept enabled Müller [76] to eliminate the possibility of nonsimple elementary divisors from Weyl's existence proof [102] somewhat to the latter's disappointment for at last he felt here was a place in applied mathematics where the elementary divisors were surely not simple.) $)^{3}$ The dissipative concept is also intimately related to the so-called optical theorem or cross-section theorem (cf. Morse and Feshbach [XI, Part II] which states that the total cross-section of a scattering process is proportional to the imaginary part of the forward scattering amplitude, a measurable quantity. Now dissipative operators are closely related to analytic functions regular in a half-plane with, say, positive-definite imaginary part and thus perhaps it is not too surprising that many of the quantities of physical interest turn out to be boundary values of an analytic function of one or more complex variables which are analytic in a suitably cut complex space. Moreover, since by Hilbert transform

\footnotetext{
2 Cf. The theory of wave propagation, New York University Notes, 1951-1952, p. I-81.

8 Nonsimple elementary divisors of course occur in the theory of systems of ordinary differential equations.
} 
theory, under suitable conditions, the real part of an analytic function can be determined from a knowledge of its imaginary part on the real line, the total cross-section is in principle determined from a knowledge of the forward amplitude. The detailed analysis of this relationship lies behind all existing dispersion theories both for potential scattering and for quantum field theory. In this connection, in the actual problems it is also necessary in many instances to perform an analytic continuation through an unphysical region to obtain a meaningful result. The successful application of a similar set of ideas to, for example, filter design is well known in network theory and has associated with it the names of Bode [II], Wiener and Lee [59].

Now dispersion relations are intimately connected with Cauchy's integral formula as well as its extension by Plemelj and in turn Cauchy's integral formula forms the basis on which one treats the resolvent of a non-self-adjoint problem since in a neighborhood of isolated singularity the resolvent operator may be under suitable conditions interpreted as a projection into the subspace characterized by the singularity. This is also intimately related to the integral form of the inverse Laplace transform, or more generally, to the resolvent of a semi-group, and thus to the initial value problem. This same set of ideas also forms the basis for the treatment of singular integral equations of Cauchy type and these in turn are essentially equivalent to those of Wiener-Hopf as we shall see. Here it will be our purpose to explore some of these interconnections and where possible give the currently best results known to us. Our emphasis will, however, be less on generality, then on the interconnections.

\section{Examples of classical self-adjoint problems exhibiting non-self-} adjoint behavior. Our first two examples will illustrate the occurrence of complex singularities, characteristic for non-self-adjoint operators, in well-set self-adjoint problems involving continuous spectra. In each case the use of the complex singularities leads to a useful and physically meaningful representation. In the first example the representation involves functions, which, while not readily interpretable in the Hilbert space of the original well-set self-adjoint problem, are readily interpretable if the corresponding initial value problem is considered. Both examples will clearly demonstrate the price paid for strict adherence to the concepts of self-adjointness.

Subsequently we will sketch the method of Ritt and Kazarinoff $[87 ; 88]$ based on the results of Sims [92] which deliberately and systematically converts a class of self-adjoint problems into a class 
of non-self-adjoint problems with resulting advantages of ease of interpretation and rigorous discussion. Thus in the area under discussion here the distinction between what really should or should not be considered a self-adjoint problem will be seen to be highly nebulous and that while the use of self-adjoint theory may be mathematically more convenient and elegant, the more general non-selfadjoint approach is often more powerful and rewarding. For want of a better term we will henceforth refer to problems of the above type as classically self-adjoint.

As a first example of a classical self-adjoint problem, let us consider a semi-infinite string, of one density between zero and one and a greater density from one to infinity, such that the end zero is held fixed. Although this example was discussed by B. Friedman in his Society talk of April, 1957, it illustrates in a simple manner, many of the salient points, and is simple enough so that a student with only a knowledge of elementary Laplace transform theory can verify the properties stated. The usual attack by the method of separation of variables will produce a self-adjoint equation in $x$, regular at the origin and of the limit-point type at infinity so that the problem is self-adjoint on $\left[L_{2}(0, \infty)\right]$ and a solution similar to a Fourier sine transform is possible in an $L_{2}$ theory if initially the string has a displacement and a velocity in the interval $(0,1)$. In this solution only continuous spectra occur. On the other hand, any physicist would argue that since we would have standing waves or an eigenfunction solution if the density from one to infinity were infinite and therefore for any density sufficiently large in this region, we would expect something like standing waves between zero and one, leaking out or spilling over and representing an outgoing wave in the region from one to infinity. This type of solution can be readily found if the initial value problem is first subjected to a Laplace transformation with respect to time and a Green's function for the resulting equation in $x$ constructed, before the inversion integral for the Laplace transform is applied. This integral can be closed by a path in the left-half plane and the singularities of the Green's function, namely the zeros of its Wronskian, investigated as a function of the Laplace parameter in which it is analytic. These are readily shown to be simple, denumerable and to lie in the left-half plane on a line parallel to the imaginary axis. At such a zero of the Wronskian, the two solutions of the differential equation from which the Green's function was constructed become linearly dependent and one has apparently found a complex eigenvalue and an associated function in contradiction to the fact that the differential equation for the Green's function, being self- 
adjoint, can have only real spectra. While the complex pole $s_{n}$ enters into the differential equation as if it were an eigenvalue, the associated functions do not satisfy the boundary condition at infinity; in fact they grow exponentially in $x$ as $x$ goes to infinity so they are not quadratically integrable and do not fall into a Hilbert space theory. ${ }^{4}$ In the physical literature these functions are often called non-modal solutions, in contrast to eigenfunction solutions which are called modal. No paradox is involved because associated with each nonmodal solution there is an exponential time factor which overrides the $x$ dependence at any point and the solution can be written with the aid of the displacement rule for the Laplace transform so that it is clear that the disturbance is zero at any point until sufficient time has elapsed for the effect of the initial disturbance in the $(0,1)$ region to arrive at the $x$ point under observation. That is, each function in $x$ vanishes outside some compact set and this supporting set grows with time. The intuitive physical picture of the physicist is therefore borne out and in addition the attenuation of the non-modal solutions is so great that only one of two of them are necessary for an excellent approximation to the phenomena involved in contrast to the representation in the right-half plane as an integral over the usual continuous spectra.

The behavior of this example is typical of many problems of mathematical physics such as the theory of $\alpha$-decay due to Gamov $[28 ; 29]$, Condon and Gurney [17]. It always occurs when there is a quasistationary or metastable state which is unstable in the long run. It therefore is an ever present part of any theory of the formation of compound nuclei and it is closely connected with so-called virtual levels. ${ }^{5}$

This type of behavior is also typical of problems involving wave propagation through inhomogeneous media as may be seen, for example, by reference to the book by Kerr [IX]. To be sure many of these

${ }^{4}$ This phenomenon when encountered in "solutions" obtained by steady-state methods which do not introduce appropriate cut-off factors is often called the "exponential catastrophe."

${ }^{5}$ For a historical sketch of the development of the method of "complex eigenvalues" as it relates to the theory of transients in continuous systems, see G. Beck and H. Nussenzveig, On the physical interpretation of complex poles of the S-matrix. I, Nuovo Cimento vol. 16 no. 3 (1960) pp. 416-449. This paper also contains an elegant discussion of the details of the phenomena sketched above for: (a) the problem of a simple harmonic oscillator attached to the extremity of a semi-infinite string; (b) the problem of electromagnetic radiation exterior to a perfectly conducting sphere and: (c) the problem of a wave packet exterior to a hard sphere in nonrelativistic quantum mechanics. 
problems involve essentially non-self-adjoint differential equations since often complex indexes of refraction occur. However, even with extra complexity, the physical phenomena do not differ significantly from that of our example which appears exceptional from this more general point of view only if self-adjointness is insisted upon. Some idea of the extra practical difficulties of computation that arise, however, can be gleaned from the article by Hartree, Michel, and Nicholson [37].

Our second example concerns the perturbation of the continuous spectrum of a real differential operator of second order considered by Titchmarsh [96]. The differential equation

$$
y^{\prime \prime}+(\lambda+\epsilon x) y=0
$$

is considered on the interval $0 \leqq x<\infty$ as a perturbation of the equation

$$
y^{\prime \prime}+\lambda y=0
$$

with the boundary condition

$$
y(0, \lambda) \cos \alpha+y^{\prime}(0, \lambda) \sin \alpha=0
$$

for $\cot \alpha>0$. (Titchmarsh notes that his previous treatment in $\S 4.1$ of $[\mathrm{XV}$, Part I $]$ is an error.)

Classically both the perturbed and unperturbed differential equation are of the limit-point type in the sense of Weyl and thus each has just one quadratically integrable solution at infinity. The problems are then classically self-adjoint. The spectrum of the unperturbed equation is continuous over $0 \leqq \lambda<\infty$ and there is one isolated negative eigenvalue. The spectrum of the perturbed operator is continuous over $\infty<\lambda<\infty$. The physical problem can be interpreted as the interaction of a photon with an atom having one bound state. The resolvent of the perturbed problem exists in the upper half of the complex $\lambda$-plane but if it is analytically continued to the lower half-plane, it has a complex pole near to the real pole of the resolvent of the unperturbed operator but just below the real $\lambda$-axis. The effect of this pole may be interpreted by saying the perturbed continuous spectrum has a resonance or is highly concentrated on the real axis in a neighborhood above this point. This example, like the first, has the advantage of being completely calculable and still typical of a large class of problems, namely resonance calculations associated with compound nuclei. In fact, one of the fundamental methods used by Heitler [VI], consists of approximating to a resonance in the continuous spectrum by a function which can be analytically continued into the lower 
half-plane. While this can be justified in special examples like the above, there is no operator theory for the general problem.

The type of behavior exhibited by our two examples is perhaps best understood by examining in somewhat more detail the relationship that exists between the usual limit-point theory and the Sommerfeld radiation condition. Limiting consideration to the perturbed problem considered by Titchmarsh, the general theory tells us that there is a uniquely defined resolvent operator $R_{\lambda}=R_{\lambda}^{+}$for $\operatorname{Im} \lambda>0$ for which no boundary condition need be specified at infinity. Since the general theory also implies that the problem is self-adjoint, the relation $\left(R_{\lambda}\right)^{*}=R_{\lambda}^{-}$can be used to define an operator $R_{\lambda}^{-}$for $\operatorname{Im} \lambda<0$ (cf. Achieser-Glasmann [I]). The operators $R_{\lambda}^{+}, R_{\lambda}^{-}$are not the same nor are they analytic continuations of each other. The expansion theory and spectral representation can be obtained by consideration of the integral

$$
\frac{1}{2 \pi i} \int_{C} R_{\lambda} d \lambda
$$

where the curve $\mathrm{C}$ encloses the real spectrum and where $R_{\lambda}=R_{\lambda}^{+}$ along its upper branch and $R_{\lambda}=R_{\lambda}^{-}$along its lower branch. From this one can obtain an expansion which is similar to that of the Fourier integral theorem. Instead of doing this, however, here the operator $R_{\lambda}^{+}$can be continued to the lower half-plane where it will represent an operator different from $R_{\lambda}^{-}$and itscomplex singularities used to give a useful representation of the continuous spectrum. This situation is in marked contrast to that for a limit circle situation where to obtain a self-adjoint problem suitable contractions in the form of boundary conditions must be imposed to allow the given operator to be suitably extended. Once this has been done, the resulting resolvent is a compact operator possessing only discrete real spectrum, thus its resolvent exists everywhere except for a definite set on the real axis and consideration of its contour integral can be restricted to small circles enclosing the real discrete spectra.

The distinction between $R_{\lambda}^{+}$and $R_{\lambda}^{-}$is important for there is an important class of non-self-adjoint operators, that of so-called dissipative operators, which admit a representation of the form (cf. $[24 ; 33])$, and $\$ 7$,

$$
\left(R_{\lambda}^{+} \phi, \phi\right)=\int_{-\infty}^{\infty} \frac{d \omega(t, \phi)}{t-\lambda}
$$

for $\operatorname{Im} \lambda>0$. 
Since little if any information is known about the equivalent of $R_{\lambda}^{-}$ for these problems in general, it becomes highly desirable to have a theory permitting the analytic continuation of the above representation to the lower half-plane. Since the function

$$
f(\lambda)=\left(R_{\lambda}^{+} \phi, \phi\right)
$$

has the properties that $f(\lambda)$ is analytic in $\lambda$ for $\operatorname{Im} \lambda>0$

$$
\operatorname{Im} f(\lambda)>0, \quad \text { for } \operatorname{Im} \lambda>0,
$$

and

$$
\sup _{y \rightarrow \infty}|i y f(i y)|<\infty, \quad \text { for } \operatorname{Im} \lambda>0,
$$

in common with the corresponding expressions for self-adjoint operators it is first necessary to solve the continuation problem for this class of analytic functions. This has been done by D. S. Greenstein [35] who proved the following:

THEOREM. If $f(z)$ is analytic such that

$$
\sup _{y \rightarrow \infty}|i y f(i y)|<\infty \text {, }
$$

and

$$
f(\lambda)=\int_{-\infty}^{\infty} \frac{d \gamma(t)}{t-\lambda}
$$

then $f(\lambda)$ can be continued across the interval $(a, b)$ if and only if the function $\gamma(t)$ is real analytic in $(a, b)$. When this is true the continuation is given by

$$
f(\lambda)=f^{*}\left(\lambda^{*}\right)+2 \pi i \gamma^{\prime}(\lambda), \quad \operatorname{Im} \lambda<0 .
$$

It should be noted that the continuation obtained agrees with the formulas of Plemelj (cf. [XII]). As a concrete illustration of the point involved, consider the function $w=-1 /(\lambda+i)$ which is analytic in the upper half-plane with positive imaginary part and which is its own analytic continuation to the lower half-plane with a singularity at $\lambda=-i$. It is easy to verify that this function admits the representation

$$
\frac{-1}{\lambda+i}=\int_{-\infty}^{\infty} \frac{d t}{\pi\left(1+t^{2}\right)} \frac{1}{t-\lambda}, \quad \operatorname{Im} \lambda>0,
$$

so that the singularity in the function is seen to be contained in the 
weight function $1 / \pi\left(1+t^{2}\right)$. In this case it is also trivial to verify that Greenstein's continuation formula reproduces $w$ in the lower half-plane.

The corresponding problem for operators is open even in the selfadjoint case where the representation corresponding to the above may be written in terms of the resolution of the identity as

$$
\left(R_{\lambda} \phi, \phi\right)=\int_{-\infty}^{\infty} \frac{\left\|E_{t} \phi\right\|^{2}}{t-\lambda} .
$$

There is an intimate relation between the operators $R_{\lambda}^{+}$and $R_{\lambda}^{-}$, the Sommerfeld radiation condition and the Plemelj formulas. The Sommerfeld radiation condition for the problem under consideration would read, for real $\lambda=k^{2}, \lim _{x \rightarrow \infty}(d y / d x)-i k y=0$ and it in effect singles out, in this case $R_{\lambda}^{+}$, the appropriate resolvent operator. More explicitly, the Green function which can be constructed through its use is the kernel of the operator $R^{+}$in the limit as $\operatorname{Im} \lambda \rightarrow 0^{+}$. Thus approaching the axis from above corresponds to an outgoing wave, from below, to an incoming wave. Furthermore, no contradiction is involved in the use of this non-self-adjoint boundary condition for a self-adjoint problem since the resolvent of self-adjoint problem is not self-adjoint for $\operatorname{Im} \lambda \neq 0$ but in fact satisfies the relation $\left(R_{\lambda}\right)^{*}=R_{\lambda}^{-}$, where the bar denotes the complex conjugate. ${ }^{6}$

The rigorous theory of perturbation problems involving continuous spectrum such as the example of Titchmarsh is far from complete. From the point of view of operator theory there is the general paper of Friedrichs [27] who considers a simplified model involving the energy of an atom in the presence of an electro-magnetic field. For this case he derives the Wigner-Weisskoff $[108 ; 109]$ formula for the natural width of the spectral lines as an approximation to the correct asymptotic behavior. For ordinary differential equations Titchmarsh $[\mathrm{XV}$, Part II] has elaborated on the type of behavior exhibited by the above example and sketched the beginnings of a similar theory for a class of partial differential equations.

While from an overall point of view, many of the problems we are discussing can be viewed as problems of perturbation theory, in many instances there appears to be no reasonable parameter, such as the fine-structure constant of quantum electrodynamics, on which to base a perturbation theory so that a fresh look at these problems as non-self-adjoint $a b$ initio rather than as bounded or unbounded per-

- For a similar discussion in more complicated situations, compare E. Gerjuoy, Outgoing boundary condition in rearrangement collisions, Phys. Rev. vol. 109 (1958) pp. 1806-1814. 
turbations of self-adjoint problems can lead to interesting new mathematics and also to useful results. ${ }^{7 \mathrm{a}}$ We will give specific illustrations in the sequel. These problems are more complicated than our example even for quantum mechanics since in many the complex-energy plane must be treated as at least a two-sheeted Riemann surface for $k^{2}=E$ and the singularities on the second sheet interpreted, for example as virtual or semi-stable states, although the interpretation is not uniform (cf. Humblet [42] and $\mathrm{Ma}$ [70]) and a general theory is lacking though results are known in special cases (cf. Flugge and Marshall [V, Aufgabe 13]; also Peierls [81]).

3. Examples of non-self-adjoint physical problems. While it is generally claimed that all quantum mechanical problems are self-adjoint, the time scale involved, or the difficulties of calculation of ten make it convenient to sub-divide the problem into pieces which may not be self-adjoint. A simple physical illustration of this is furnished by Bohr's idea of the compound nucleus. Here he showed that in a collision between two nuclei, at least one of which is heavy, an unstable compound nucleus will be formed and that the life-time of such a compound nucleus on a nuclear scale will be very large. Further such nuclei have fairly well-defined energy levels (usually referred to as "virtual" or "resonance" levels) and the positions of these levels and the properties of the associated metastable states determine the crosssections of the nuclear reactions which may occur. By using a perturbation theory in which certain interaction terms in the Hamiltonian describing the system were neglected, Breit and Wigner derived in 1936 a formula for the probability of a nuclear reaction in the presence of one virtual state and their work was generalized by Bethe [1], to whom we refer the reader for the historical development, to take into account all possible virtual states. In these theories the perturbation theory was carried out only to the second order of approximation. In an attempt to overcome this last limitation, Peierls and Kapur [82] developed a formalism involving complex eigenvalues and complex states. In particular, they argued that if the resonance levels in the compound nucleus are narrow so that the escape of a particle is a rare event, the states of the compound nucleus will undergo very little change if the particle is prevented from escaping altogether by the imposition of a suitable restriction on the wave function outside the nucleus. They then perturbed this "boundary condition" in order

\footnotetext{
7a Several recent examples are to be found in K. O. Friedrichs, Perturbation of spectra in Hilbert space, Lecture Notes, Boulder Seminar in Applied Mathematics, Summer, 1960.
} 
to obtain an approximate solution to the problem. We will now describe their procedure for the simplest case which involves zero angular momentum and refer the interested reader to their paper, and those of Humblet [41] and Brown [8] for the subsequent developments and the general case. Under the assumption that only one particle scattering is involved in a central force field of finite extent, the partial wave $(l=0)$ satisfies the wave equation (in appropriate units)

$$
\frac{d^{2} \phi}{d r^{2}}+[E-V(r)] \phi=0, \quad \phi(r=0)=0,
$$

in which $V(r)$ is the potential energy, $E$ the energy of the particle. For $r \geqq r_{0}, V$ vanishes, and the above equation reduces to

$$
\frac{d^{2} \phi_{E}}{d r^{2}}+k^{2} \phi_{E}=0
$$

where the wave vector $k$ is defined by $k^{2}=E$. The solution of (3.2) can be written for $r \geqq r_{0}$ as

$$
\phi_{E}=\frac{I}{k} \sin k r+S e^{i k r},
$$

where $I$ is the amplitude of the incident wave and $S$ that of the scattered wave. Since

$$
\frac{d \phi_{E}}{d r}=I \cos k r+i k S e^{i k r},
$$

it is evident that

$$
I e^{-i k r_{0}}=\left.\frac{d \phi_{E}}{d r}\right|_{r_{0}}-i k \phi_{E}\left(r_{0}\right)
$$

and

$$
S=\cos k r_{0} \phi_{E}\left(r_{0}\right)-\frac{1}{k} \sin k r_{0}\left(\frac{d \phi_{E}}{d r}\right)_{r_{0}} .
$$

Thus it is evident that when there is no incident wave, one should have

$$
\frac{d \phi}{d r}-i k \phi=0, \quad \text { at } r=r_{0} .
$$

While this condition is obviously incompatible with the original one can consider the auxiliary problem 


$$
\frac{d^{2} \phi_{n}}{d r^{2}}+\left[W_{n}-V\right] \phi_{n}=0, \quad W_{n} \neq E
$$

with the boundary conditions (3.3) and (3.4) where $k$ is a fixed constant. Now the usual arguments show that the functions defined by this problem are real orthogonal (although they are complex valued) and the corresponding eigenvalues are complex because of (3.4). Once these functions have been determined, one can see formally that any solution of (3.1) satisfying (3.3), the true boundary condition, can be expanded in the form

$$
\phi_{E}=\sum a_{n} \phi_{n}+X
$$

where $\phi_{n}$ satisfy the homogeneous condition (3.4) and $X$ the inhomogeneous condition (3.3).

A variant of this procedure has been proposed by Siegert [91] and investigated extensively by Humblet [41]. From the ratio $S / I$ given above,

$$
S / I=\left(\phi_{E}\left(r_{0}\right) \cos k r_{0}-\phi_{E}^{\prime}\left(r_{0}\right) \frac{\sin k r_{0}}{k}\right) e^{-k r_{0}} /\left[\phi_{E}^{\prime}\left(r_{0}\right)-i k \phi_{E}\left(r_{0}\right)\right]
$$

one seeks the singularities which arise from the zeros of the denominator. These singularities can be defined by the eigenvalue problem

$$
\frac{d^{2} \psi_{n}}{d r^{2}}+\left(\lambda_{n}-V\right) \psi_{n}=0
$$

with the boundary conditions

$$
\psi_{n}=0 \text { at } r=0,
$$

and

$$
\frac{d \downarrow_{n}}{d r}-i k_{n} \psi_{n}=0 \text { at } \quad r=r_{0} .
$$

This last boundary condition in contrast to (3.4) makes the compound states independent of the energy of the incoming particle and makes the result of the calculation of the scattering independent of the scale $r_{0}$ beyond which the potential is assumed to vanish. The resulting expansion may be interpreted in terms of "radio-active states," but it possesses the disadvantage that the expansion is not in terms of orthogonal functions in either a real or hermitian sense.

The third way of describing this physical process is by the method 
of Wigner-Eisenbud [110] where only real eigenvalues occur but this in turn leads to a knotty problem in analytic continuation (cf. Wigner [106], Wigner and von Neumann [111] and the review article by Lane and Thomas [58] for a more thorough investigation of the relations between these methods). From a physical point of view the method of Wigner-Eisenbud seems preferable to the case where few levels contribute to the scattering process while the method of Kapur and Peierls is still preferable even today as evidenced by the review article by G. E. Brown [8] for the many level case since the sum over levels enters linearly in the scattering amplitude. While, to paraphrase Lane and Thomas, the method of Siegert seems to be the best of all possible worlds, the occurrence of complex eigenvalues and non-orthogonality makes the application of the method to the general situation difficult.

The mathematical justification of each of these methods is problematical and probably only the method of Wigner-Eisenbud has a real mathematical basis as a result of Wigner's collaboration with von Neumann. The justification of the Kapur-Peierls method usually given is a subsequent intuitive paper by Peierls [80] in which a rigorous derivation due to Skyrme is promised. To the best of our knowledge this derivation has never appeared. In the mathematical literature only the work of J. Schwartz [90], M. Naimark [77] and Sims [92] has direct bearing. The first two of these are highly restrictive in that Schwartz treats only bounded perturbations while Naimark has severe differentiability and integrability conditions imposed on the potential. While the work of Sims is not limited to bounded perturbations, the Hilbert space approach employed by him leaves open the question of the type of behavior illustrated in our example of the semi-infinite string (cf., however, §5).

The conventional formal scattering theory provides yet another example of classical self-adjoint problems. In this formalism one seeks to relate the solution of the interaction free Schrödinger equations at time equal to plus or minus infinity with those of an interaction free Schrödinger equation. This is accomplished by noting that any solution of the free Schrödinger equation can be written in the form

$$
\Phi(t)=\int_{0}^{\infty} e^{-i E t} \phi(E) d E
$$

where $\phi$ is determined by the Fourier inversion integral. The scattering state is then given by (cf. Lippmann and Schwinger [65], GellMann and Goldberger [32] 


$$
\Psi(t)=\int_{0}^{\infty} e^{-i E} \psi(E) d E
$$

where $\psi(E)$ is the solution of

$$
\psi(E)=\phi(E)+G(E) V \psi(E),
$$

with the Green's operator representing an outgoing wave. The weak point in this argument is that the integral defining $\phi(E)$ does not have a meaning in a Hilbert space theory for it has no limit in either a weak or a strong sense. In fact $\phi(E)$ would be an eigenvector of the unperturbed Hamiltonian belonging to the value $E$ of the continuous spectrum of $H_{0}$. Several methods are used to overcome this difficulty but all are objectional on some grounds. The most customary method is the use of the "adiabatic hypothesis" which is tantamount to the introduction of a convergent factor $\exp (-\epsilon|t|$ ) (cf. Lippman and Schwinger [65]). Friedrichs [27] in another section of his basic paper has made an ingenious use of the Riemann-Lebesgue lemma and the Plemelj formulas, which incidentally are also used by Lippman and Schwinger, to eliminate the necessity for the "adiabatic hypothesis" and his approach has been developed by Moses [74]. Recently Jauch [43] has given an alternate approach to the entire theory from a rigorous Hilbert space point of view but while both the approaches by Friedrichs and Jauch are conceptually satisfying, they do not consider the problem of the solution of equation (3.5) or its equivalent. In order to have a practical theory for even potential scattering, it is necessary to solve an equation of this form, which, explicitly, in this case is:

$$
\psi(\boldsymbol{r})=e^{i \cdot \mathbf{k} \cdot \boldsymbol{r}}-\int \frac{e^{i k R}}{4 \pi R} V\left(\boldsymbol{r}^{\prime}\right) \psi\left(\boldsymbol{r}^{\prime}\right) d^{3} \boldsymbol{r}^{1}, \text { where } \lambda=k^{2}, R=\left|\boldsymbol{r}-\boldsymbol{r}^{\prime}\right| .
$$

The kernel of this equation is the free space Green's function of Helmholtz's equation which satisfies the outgoing radiation condition, or alternately in view of our remarks of $\S 2$, it is the limit of the kernel of the resolvent operator $R_{\lambda}^{+}=\left(\nabla^{2}+\lambda\right)^{-1}$ as $\operatorname{Im} \lambda \rightarrow 0^{+}$. Explicitly,

$$
\frac{e^{i k R}}{4 \pi R}=\lim _{\bullet \rightarrow 0^{+}} \frac{1}{4 \pi R} \int_{\infty}^{\infty} \frac{t \sin t R d t}{t^{2}-\left(k^{2}+i \epsilon\right)} .
$$

It is clear that this kernel is real symmetric although complex-valued and is thus certainly not the kernel of a self-adjoint operator. Alternately, since $R_{\lambda}=\left(\nabla^{2}+\lambda\right)^{-1}$ does not exist in general for $\lambda$ real and positive, and for $\operatorname{Im} \lambda \neq 0$, it has the property that $R_{\lambda}^{*}=R_{\lambda}^{-}$, the operator $R$ is also not self-adjoint for $\operatorname{Im} \lambda \neq 0$ and the "inversion in 
the continuous spectrum" can only be interpreted in terms of the above limiting process. The resolvent kernel can also be constructed by the usual procedures for Green's function for $k$ real and positive in a unique fashion only if the Sommerfeld radiation condition is imposed and the limiting operation

$$
\lim _{\operatorname{Im} \rightarrow 0^{+}} R_{\lambda}^{+}
$$

is therefore seen to be equivalent to this procedure. To reiterate, although the original problem as defined by the Schrödinger equation is self-adjoint, the treatment of a general potential case which cannot be solved explicitly involves consideration of the above non-self-adjoint operator.

Until recently no general theory existed for the solution to the above non-self-adjoint integral equation which was not formal in the sense that non-quadratically integral functions occurred. To be sure this equation has frequently been treated by methods of successive approximation which lead to Liouville-Neumann or Born series and it has also been discussed with the aid of Fredholm theory by Jost and Pais [44]. Their discussion was concerned, however, with the uniform and absolute convergence of the Fredholm series for the first iterate of the above equation and as such is still open to the above objection, and their results were too unwieldly to yield calculable results in general. Subsequently, Khuri [47] used this same method to rigorously deduce the dispersion relations for potential scattering. In order to give a rigorous $L_{2}$ theory, Dolph and Ritt [21] and Dolph and Penzlin [24] symmetrized the above integral equation for positive or negative definite potential and replaced it by an equivalent integral equation in a larger Hilbert space where it was amenable to treatment by the developing theory of non-self-adjoint dissipative operators which will be discussed in $\$ 7 . .^{7 \mathrm{~b}}$ The resulting quantities of physical interest are unaffected by this procedure which is however not general because of the assumption of definiteness of the potential. It seems reasonable to conjecture that the theory of indefinite Hilbert spaces touched upon briefly in $\$ 10$ will eventually permit the removal of this restriction.

An alternate rigorous and more complete treatment which uses the Riesz theory of completely continuous operators in a Banach space has just been published by Ikebe [115] who in turn leans heavily on

7b Note added in proof. Cf. the treatment by H. Rollnik, Streumaxima und gebundene zustände, Z. Physik vol. 145 (1956) p. 639, where a physical interpretation of the complex eigenvalues of this theory is given. 
some earlier work of Povzner [116]. In this theory it is assumed that the potential $V(x)$ is a real-valued function which is locally Hölder continuous except for a finite number of singularities that $V(x)$ is in $L_{2}\left(E^{3}\right)$ and of order $0\left(|x|^{-2-h}\right)$ at infinity for $h>0$. It is known that these conditions are sufficient to guarantee the unique self-adjoint extensions $H$ and $H_{0}$ of the operators $-\nabla^{2}+V(x)$ and $-\nabla^{2}$, respectively, such that the domains of $H$ and $H_{0}$ will be equal (cf. Kato [117]). The first step consists in establishing that the resolvent $(H-\lambda I)^{-1}$ is an integral operator of the Carleman type after which the Banach space $B$ of all continuous functions $u(x)$ defined on $E^{3}$ which tend to zero uniformly as $x$ tends to infinity is introduced with the norm $\|u\|_{B}=\max _{x \in E^{3}}|u(x)|$. In terms of the functions

$$
p(r, k)=-\frac{1}{4 \pi} \int \frac{e^{i k R}}{4 \pi R} V\left(r^{\prime}\right) e^{i k \cdot r^{\prime}} d^{3} r^{\prime}
$$

and

$$
\phi(r, k)=\psi(r)-e^{i k \cdot r}
$$

the scattering integral equation can be written as

$$
\phi(r, k)=p(r, k)-\frac{1}{4 \pi} \int \frac{e^{i k R}}{R} V\left(r^{\prime}\right) \phi\left(r^{\prime}, k\right) d^{3} r^{\prime}
$$

and readily discussed in $B$ since it can be shown that $p$ is in $B$. In particular the integral operator which occurs in the above expression is of the Hilbert-Schmidt type and therefore defines a completely continuous operator which is an extension of $\left(H_{0}-\lambda\right)^{-1} V$. In particular for $\operatorname{Im} k \geqq 0$, this operator takes $B$ into $B$ and the Fredholm alternative applies. The connection with the $L_{2}$ theory which is of final interest is obtained by observing that for any $f$ in $B, V f$ is in $L_{2}\left(E^{3}\right)$. Thus, for example, if for $\operatorname{Im} \lambda \neq 0, \operatorname{Im} k>0$,

$$
f=-\left(B_{0}-\lambda\right)^{-1} V f
$$

for $f$ in $B$, then $f$ is in the domain of $H_{0}$, and application of $\left(H_{0}-\lambda\right)$ to both sides yields

$$
\left(H_{0}-\lambda\right) f=-V f
$$

or

$$
H f=\lambda f
$$

which shows that $f \equiv 0$ since $H$ is self-adjoint and $\lambda$ is not real. In addition, Ikebe proves that there is no continuous spectrum of $H$ on the negative real axis, that there are no positive eigenvalues, that 
the negative eigenvalues if they exist are discrete, of finite multiplicity and have no limit point other than zero, and that the spectrum on the positive real axis is absolutely continuous. Furthermore he develops a generalized Fourier transform theory which utilizes the continuous set of distorted plane wave functions obtained from the solution to the scattering integral equation in conjunction with the eigenfunctions corresponding to negative real eigenvalues should they exist. Finally he gives an alternate proof of the theorem of Kuroda [118] which states that the $S$ matrix is unitary if the potential $V$ as defined above has the additional property of being in $L_{1}\left(E^{3}\right)$ $\cap L_{2}\left(E^{3}\right)$.

To return to the initial remark of this section, the situation in regard to the scattering integral equation is quite typical of the procedure used in many similar problems whenever it is reasonable to decompose the Hilbert space of quantum mechanics into two orthogonal subspaces $\mathfrak{H C}_{1}$ and $\mathfrak{F C}_{2}$ with associated projection operators $P_{1}$ and $P_{2}$. Under these circumstances there will be an associated decomposition of the Hamiltonian $H$ of the total problem into the form

$$
H_{1}=H_{11}+H_{12}+H_{21}+H_{22}
$$

where

$$
H_{i k}=P_{i} H P_{1}, \quad(i k=1,2) .
$$

Under this decomposition, the Schrödinger equation will take the form

$$
\begin{aligned}
& i \frac{\partial \psi_{1}}{\partial t}=H_{11} \psi_{1}+H_{12} \psi_{2}, \\
& i \frac{\partial \psi_{2}}{\partial t}=H_{21} \psi_{1}+H_{22} \psi_{2} .
\end{aligned}
$$

Now if one can solve the problem

$$
\left(i \frac{\partial}{\partial t}-H_{11}\right) U(t)=0, \quad U(0)=P,
$$

the first of these equations can be written as

$$
\psi_{1}=U\left(t-t_{0}\right) \psi_{1}\left(t_{0}\right)-i \int_{t_{0}}^{t} U\left(t-t^{\prime}\right) H_{12} \psi_{2}\left(t^{\prime}\right) d t^{\prime}
$$

and if this is introduced into the second in the presence of an initial condition, one obtains the integro-differential equation 


$$
i \frac{\partial \psi_{2}}{\partial t}=H_{22} \psi_{2}-i \int_{t_{0}}^{t} H_{21} U\left(t-t^{\prime}\right) H_{12} \psi_{2} d t^{\prime} .
$$

After a Fourier transform this becomes

$$
E \psi_{2}(E)=\left\{H_{22}-H_{21} * G(E) * H_{12}\right\} \psi_{2}(E)
$$

where $G(E)$ is the formal Fourier transform of $U(t)$ and in general it will involve the non-self-adjoint operator

$$
H_{22}-H_{21} * G(E) * H_{12} .
$$

Specific instances of the use of this decomposition can be found implicitly in Bethe [1] and in Dolph and Penzlin [24].

In contrast to the above, problems of mathematical physics involving transport phenomena are often essentially non-self-adjoint.

One of the best known example of this is furnished by the OrrSommerfeld equation for the perturbation of linearized flows involving a viscous fluid. In the case of plane Poiseuille flow it takes the form

$$
\nu\left(f^{\mathrm{iv}}-\alpha^{2} f^{\prime \prime}+\alpha f\right)=i \alpha\left[\left(1-y^{2}\right)-c\right]\left[f^{\prime \prime}-\alpha^{2} f+2 f\right]
$$

with the boundary conditions

$$
f( \pm 1)=f^{\prime}( \pm 1)=0 .
$$

This equation arises from the linearized time dependent partial differential equations when the assumption

$$
\psi(x, y, t)=e^{i \alpha(x-c t)} f(y)
$$

is made for the perturbed stream function. The curve of neutral stability is described as the locus where $\operatorname{Re} \alpha=0$ as a function of viscosity $\nu$ or, more usually, Reynolds number. The question as to whether unstable modes exist has had a long history and was not settled affirmatively in this case before the work of Lin [VIII] because of the mathematical complexity of the asymptotic theory involved. In this connection in order to further support our contention that there are no firm criteria dictating the use of self-adjoint theory or non-self-adjoint theory, it should be noted that Hopf [39] used selfadjoint methods to establish the existence of a weak solution to the Navier-Stokes equation valid for all time and that Dolph and Lewis [22] made an application of his ideas to discuss the same phenomena governed by the Orr-Sommerfeld equation. In this method, the OrrSommerfeld equation was treated with the aid of a self-adjoint equation which possessed a known expansion theorem and the problem 
was thereby reduced to the consideration of an infinite set of coupled ordinary differential equations for the unknown expansion coeffcients as functions of time. A $20 \times 20$ truncation of the resulting system reproduced the nose of the stability curve to a high order of approximation. Recent developments in this theory include proof of the completeness theorem for the non-self-adjoint expansions of the Orr-Sommerfeld equation due to Schensted [89] and the demonstration by Case $[12 ; 14]$ of the existence of a class of distribution solutions in the case of the inviscid limit which are limits of viscous solutions. As a result of this work, it is now possible to make the transition from the Navier-Stokes equations to the Euler equations in a manner reminiscent of the passage from quantum to classical mechanics.

For the problem of neutron diffusion, Lehner and Wing $[60 ; 61]$ considered a non-self-adjoint spatial operator defined by the timeindependent equation; namely, the operator

$$
A f=\mu \frac{\partial f}{\partial x}+\frac{1}{2} c \int_{-1}^{1} f\left(x, \mu^{1}\right) d \mu^{1} .
$$

This operator is densely defined in a subset of the Hilbert space of measurable function such that the integral

$$
\int_{-a}^{\infty} d x \int_{-1}^{1}[f(x, \mu)]^{2} d \mu
$$

exists. The point spectrum of it consists of a finite nonempty set of real numbers and it has an empty residual spectrum. On the other hand, they also showed that the continuous spectrum consisted of an entire half-plane, thus in (61) their expansion theorem involved consideration of a contour integral at infinity which could, practically, be evaluated only approximately. The above operator is particularly interesting in that the nature of its spectrum is highly sensitive to the domain of definition considered. Thus some as yet unpublished work of $\mathrm{K}$. Friedrichs and J. Lehner demonstrates that replacing $-a$ by $-\infty$ in the above double integral is sufficient to confine the continuous spectra to an axis as in the self-adjoint case. For infinite or semi-infinite regions Case [11] was able to replace the above $L_{2}$ theory by an $L_{1}$ theory in which he was able to determine the class of elementary time-independent solutions and obtain both half- and full-range completeness theorems in terms of distributions. The approach of Case's was inspired by earlier work of Van Kampen $[98 ; 99]$ and depends heavily upon the use of techniques from the theory of 
singular integral equations. A brief description of some of the ideas will be given in $\$ 8$.

4. Singular non-self-adjoint Sturm-Liouville problems. Chapter 12 of Levinson and Coddington [IV] furnishes an introduction to regular non-self-adjoint problems on a finite interval. A related result is that due to Friedman and Mishoe [26] which explicitly is:

TheOREM 4.1. Let $F(x)$ be a function of bounded variation $0 \leqq x \leqq 1$ and let $u_{n}(x)$ be the eigenfunctions of the system $(A+\lambda B)=0, u(0)$ $=u(1)=0$ where $A$ is the operator

$$
\frac{d^{2}}{d x^{2}}+q(x)
$$

and $B$ is the operator

$$
-\frac{d}{d x}+P(x)
$$

Furthermore let $v_{n}(x)$ be the eigenfunctions of the system adjoint to $(A+\lambda B)=0, u(0)=u(1)=0$ and let $C(\lambda) e^{\lambda x}$ be the Wronskian of the equation $(A+\lambda B)=0$. If $P(\xi, x)=\int_{\xi}^{x} p(t) d t$ and

$$
F(0+)+\exp [-P(0,1)] F(1-)=0,
$$

then the series

$$
\sum_{\infty}^{\infty} u_{n}(x) \int_{0}^{1} F(\xi)\left\{\frac{p(\xi) v_{n}(\xi)+v_{n}^{\prime}(\xi)}{c^{\prime}\left(\lambda_{n}\right)}\right\} d \xi
$$

converges to $F(x)$ at every point where $F(x)$ is continuous in $0<x<1$. At all other points it converges to $F(x+0) / 2+F(x-0) / 2$. If $F(x)$ does not satisfy the boundary conditions (4.1) then the series converges to:

$$
\begin{aligned}
\frac{1}{2} F(x+0)+ & \frac{1}{2} F(x-0) \\
& -\frac{1}{2} \exp P(0, x\{F(0+)+\exp [-P(0,1)] F(1-)\} .
\end{aligned}
$$

While the singular self-adjoint Sturm-Liouville problem has been extensively treated by Weyl [101], Stone [XIV], Titchmarsh [XV, Parts I-II), and Kodaira [46], Phillips [83] appears to be the first to treat the corresponding problem for complex boundary conditions. Later Sims [92] was able to generalize the treatment by Phillips, but in the interim J. Schwartz [90] treated the special case of a bounded 
perturbation on a finite interval by the use of Dunford's theory of spectral operators. ${ }^{8} \mathrm{His}$ result of interest to us here is:

THEOREM 4.2. Let $T$ be the unbounded differential operator defined by the formal differential operator

$$
-\frac{d^{2}}{d x^{2}}
$$

and the boundary conditions

$$
\begin{aligned}
& f(0)-k_{0} f^{\prime}(0)=0, \\
& f(1)-k_{1} f^{\prime}(1)=0,
\end{aligned}
$$

where $k_{0}$ and $k_{1}$ are arbitrary, possibly infinite, complex numbers. Then if $B$ is an arbitrary bounded operator $T+B$ is a spectral operator and every function $f$ in $L_{2}(0,1)$ can be expanded in a series of eigenfunctions (including possibly, a finite number of solutions of equations of the type $\left.(T+B-\lambda)^{k} f=0\right)$ of $T+B$ which converges unconditionally in the topology of $L_{2}$ (cf. also Lidskit [63]).

Sims allowed the interval to be infinite and generalized the limitcircle, limit point approach of Weyl [101] to complex $q(x)$ (cf. Chapter 9 of [IV]). One begins as in the self-adjoint singular theory by considering a fundamental set of solutions of the equation

$$
-y^{\prime \prime}+[q(x)-\lambda] y=0 \text { for } \operatorname{Im} q(x) \leqq 0 \text { and } \operatorname{Im} \lambda \geqq 0
$$

which are normalized by

$$
\begin{aligned}
\phi\left(x_{0}, \lambda\right) & =\sin \alpha_{0}, & \theta\left(x_{0}, \lambda\right) & =\cos \alpha_{0}, \\
\phi^{\prime}\left(x_{0}, \lambda\right) & =-\cos \alpha_{0}, & \theta^{\prime}\left(x_{0}, \lambda\right) & =\sin \alpha_{0} \text { for } \operatorname{Im} \alpha_{0} \leqq 0 .
\end{aligned}
$$

The boundary condition

$$
\alpha \psi(\phi, \lambda)+\beta \psi^{\prime}(b, \lambda)=0, \alpha, \beta \text { complex }
$$

is imposed on the combination

$$
\psi(x, \lambda)=\theta(x, \lambda)+m(\lambda, b) \phi(x, \lambda) .
$$

Then, as in Weyl's theory, if $z=\alpha / \beta$

$$
m=-\frac{\theta(b, \lambda) z+\theta^{\prime}(b, \lambda)}{\phi(b, \lambda) z+\phi^{\prime}(b, \lambda)}
$$

is a nonsingular linear fractional transformation which maps the

- The results of Schwartz have been generalized to higher order differential equations by H. Kramer, Perturbations of differential operators, Pacific J. Math. vol. 7 no. 3 (1957) p. 1405. 
lower $z$-plane into a circle $C_{b}(\lambda)$ in the $m$-plane and if $b$ is allowed to approach infinity (or any singular point of the differential problem) a limit circle or limit point is shown to exist. The radius of the circles $C_{b}(\lambda)$ is given by

$$
r_{b}(\lambda)=\left\{-\sinh 2 \operatorname{Im}\left(\alpha_{0}\right)+2 \int_{x_{0}}^{b}[\operatorname{Im} \lambda-\operatorname{Im} q(x)]|\phi|^{2} d x\right\}^{-1}
$$

and furthermore in all cases the integral

$$
\int_{x}^{\infty}\left|\psi_{\infty}(x, \lambda)\right|^{2}[\operatorname{Im}(\lambda)-\operatorname{Im} q] d x
$$

exists and hence a fortiori the integral

$$
\int_{x}^{\infty}\left|\psi_{\infty}(x, \lambda)\right|^{2} d x
$$

exists. There are now three possible cases at each singular point; namely, (1) (4.4) is the only square integrable solution of (4.1). This is a limit point case since the radius defined by (4.2) will tend to zero; (2) there are two linearly independent solutions to (4.1) which are square integrable in $\left(x_{0}, \infty\right)$ but only one solution which is square integrable with respect to the weight function $\operatorname{Im}(\lambda)-\operatorname{Im} q(x)$. This again is a limit point case; (3) There exist two linearly independent solutions square integrable on $\left(x_{0}, \infty\right)$ with respect to the weight function $\operatorname{Im}(\lambda)-\operatorname{Im} q(x)$, hence, a fortiori, square integrable with respect to the unit weight function. This is a limit circle case. For the doubly infinite interval there are six distinct cases in all, but for all of them we have the following:

THEOREM 4.3. The resolvent $R(\lambda)$ exists as a linear bounded operator defined for all of $L_{2}(a, b)$ for $\operatorname{Im}(\lambda)>0$ and the resolvent set contains the upper half of the $\lambda$-plane. Furthermore,

and

$$
\|R(\lambda)\| \leqq 1 / \operatorname{Im} \lambda,
$$

$$
\int_{a}^{b}|R(\lambda) f|^{2}[\operatorname{Im} \lambda-\operatorname{Im} q(x)] d x<\infty .
$$

The range of the resolvent is the domain defined by all functions in $L_{2}(a, b)$ such that $f, f^{\prime}$ are absolutely continuous, $-f^{\prime \prime}+q f$ is in $L_{2}(a, b)$, and $W_{b}\left[\psi_{1}, f\right]=W_{a}\left(\psi_{0}, f\right)=0$ for the limiting functions $\psi_{0}, \psi_{1}$, defined by the limiting process. This same set is also the domain of the operator $-d^{2} / d x^{2}+[q(x)-\lambda]$ and the range of this operator is $L_{2}(a, b)$.

In addition, one has the 
THEOREM 4.4. In cases $(2,2),(2,3)$ and $(3,3)$ the spectrum of the differential operator defined in the above domain is a pure point spectrum consisting of discrete points in the lower-half plane. The corresponding resolvent is a completely continuous operator.

Sims proceeds to investigate the analytic behavior and the possible contractions associated with the domain of this operator. In particular, he determines a well-set problem in which the behavior of the functions depends on a continuous boundary condition but for the details we shall have to refer the interested reader to his paper. ${ }^{9}$

5. From the self-adjoint to the non-self-adjoint. Even before the theory of Sims was developed, Marcuvitz [73] discussed, somewhat formally to be sure, several interesting special spectral representations of singular Sturm-Liouville problems which were often non-selfadjoint. In particular for the differential equation

$$
\frac{d^{2} y}{d r^{2}}+\left(k^{2}+\lambda / r^{2}\right) y=0
$$

subject to the boundary conditions $d y / d r=0$ at $r=r_{1}$ and $\lim _{r \rightarrow \infty} d y / d r$ $-i k y=0$ he noted that a unique Green's function $G$ could be constructed for $k$ real and $\operatorname{Im} \lambda>0$ and that under these conditions the problem was like a "limit circle" self-adjoint problem in that two linearly independent square integrable solutions existed from $r_{1}$ to infinity. He also noted that if $k$ were taken complex with $\operatorname{Im} k>0$, the problem became of the limit point type and the radiation condition could be replaced by the simpler requirement that $G$ must tend to zero as $r$ tends to infinity.

While a precise meaning can now be assigned to these statements as a result of the theory of Phillips and Sims which Marcuvitz in part stimulated, the above observation of his has been used systematically by Ritt and Kazarinoff $[87 ; 88]$ to develop a theory of scattering for the scalar wave equation which depends upon the theory of Sims for the separated ordinary non-self-adjoint differential equations which occur. This approach has the advantage of requiring only consideration of a limit point situation and in addition the Sommerfeld radiation condition is replaced by the more natural requirement of quadratic integrability. It is also interesting in that through use of a

- Recent related results have been announced by V. A. Marcenko and F. S. RofeBeketov in Dokl. Akad Nauk. SSSR, vol. 130 (1958) pp. 963-966, in a paper entitled Expansion in characteristic functions of non-self-adjoint singular differential operators, MR 2092, vol. 21 no. 4 (1960) p. 400. 
Tauberian and an Abelian theorem, the relationship between the steady state method and the associated initial value problem is made clear.

More specifically, the steady state exterior problem for the Helmholtz equation involves finding a solution of the equation

$$
\left(\nabla^{2}+w^{2}\right) \psi=\rho(x)
$$

in which $\rho(x)$ represents a spatial source distribution vanishing outside a compact set. In this equation $w$ is the frequency of radiation and the problem becomes well set if $\psi$ is subject to a boundary condition appropriate to the first, second, or third boundary value problems of potential theory on the surface of the scattering body and to the Sommerfeld radiation condition

$$
\lim _{R \rightarrow \infty} \int\left|\frac{\partial \psi}{\partial r}-i w \psi\right|^{2} d S=0 .
$$

This problem arises from the corresponding time dependent wave equation

$$
\nabla^{2} u=u_{t t}=\rho(x) e^{i w t}
$$

when the hypothesis is made that solutions can be represented in the form

$$
u(x, t)=\psi(x) e^{i w t} .
$$

This problem is however not well set until initial value for $u(x, t)$ and $u_{t}(x, t)$ are prescribed and once this has been done the above representation may no longer be justified.

To resolve this difficulty and to determine in what sense the steady-state approach is valid, Ritt and Kazarinoff start with the initial value problem and prove the following two theorems. (The symbol $x$ will stand for the point $(x, y, z)$ in $E^{3}$.)

THEOREM 5.1. If $u$ is a solution for $t>0$ to

$$
\nabla^{2} u-u_{t t}=\rho(x) e^{i w t}
$$

subject to $\partial u / \partial n=0$ on the boundary of a region $B$ and

$$
\begin{gathered}
u(x, 0)=f(x), \\
u_{t}(x, 0)=g(x),
\end{gathered}
$$

then $u$ is unique and if $f, g$, and $\rho$ are zero for $|x|>R$, then $u(x, t)=0$ for $|x|>R+t$. 
TheOREM 5.2. Let $V$ denote the closure of the region exterior to $B$ and let $v(x, t)=e^{-i w t} u(x, t)$, where $u(x, t)$ is the solution of Theorem (5.1). Then:

(a) The Tauberian limit

$$
v^{*}(x, t)=\lim _{T \rightarrow \infty} \frac{1}{T} \int_{0}^{T} v(x, t) d t
$$

exists and

(b) $v^{*}(x, t)$ satisfies the Helmholtz equation

$$
\left(\nabla^{2}+w^{2}\right) v^{*}=\rho(x),
$$

the boundary condition $\partial v^{*} / \partial u=0$ on $B$ and the Sommerfeld radiation condition.

(c) The Laplace transform $\hat{v}(x, s)$ of $v(x, t)$ exists in $L_{2}(V)$ for $s>0$.

(d) The Abelian limit $\left(\lim _{s \rightarrow 0+} s \hat{v}(x, s)\right.$ exists and

$$
\lim _{s \rightarrow 0+} s \hat{v}(x, s)=v^{*}(x) \text {. }
$$

(e) If $\phi(x, s)=s \hat{v}(x, s)$ then $\phi(x, s)$ satisfies the equation

$$
\left[\nabla^{2}+(w-i s)^{2}\right] \phi(x, s)=\rho(x)
$$

and the conditions that $\partial \phi / \partial u=0$ on $B$ and $\phi$ is in $L_{2}(V)$.

Thus the steady-state method can be properly applied to the problem given in (e) above and when this is done for a separable problem the resulting ordinary differential equations will be non-self-adjoint and precisely of the form treated by the Sims theory. By working with the resolvents of the separated equations one is then able to obtain a solution to the original scattering problem and completely justify procedures used by Marcuvitz [73] and Sommerfeld [XIII].

The replacement of the Sommerfeld radiation condition by that of quadratic integrability is not mysterious when one recalls that Wilcox [112] has shown that the Sommerfeld radiation condition in the form

$$
\lim _{R \rightarrow \infty} \int\left|\frac{\partial \phi}{\partial r}+i(w-i s) \phi\right|^{2} d S=0
$$

implies the existence of

$$
\int_{R}^{\infty}|\phi(x, s)|^{2} d x
$$

for $s>0$. Recently in a letter to Ritt, he has demonstrated the converse so that for $s>0$, these two are completely equivalent. Recently 
Wilcox [113] has also examined the relationship between the steadystate problem and the initial value problem from a somewhat different point of view and given an excellent account of the equivalence relations existing between the different forms of the radiation condition.

The work of Ritt and Kazarinoff is then a specific instance where the theory of non-self-adjoint operators has materially helped our understanding and placed a large part of existing scattering theory on a firm foundation.

It is interesting to observe that Titchmarsh [97; XV, Part II] encountered non-self-adjoint ordinary differential operators in his treatment of the hydrogen atom in an electric field. Specifically this occurs for the problem

$$
\nabla^{2} \psi+\left(\lambda+\frac{a}{r}-2 b z\right) \psi=0, \quad b>0
$$

which, after the transformation of Schrödinger, separates into the differential equations

$$
\begin{gathered}
x^{\prime \prime}(u)+\left[\tau+\lambda u^{2}-b u^{4}-\left(n^{2}-\frac{1}{4}\right) u^{-2}\right] x(u)=0, \\
w^{\prime \prime}(v)+\left[\tau+2 a+\lambda v^{2}+b v^{4}-\left(n^{2}-\frac{1}{4}\right) v^{-2}\right] w(u)=0,
\end{gathered}
$$

where $\tau$ becomes the eigenvalue parameter and $\lambda$ is complex. After observing that the method of Weyl guarantees the existence of Green's functions $g(u, s, \tau, \lambda)$ and $\gamma(v, t, \tau, \lambda)$ of (5.1) and (5.2) respectively for real $\lambda$ only, he directly constructs these functions which are meromorphic with, for example, the poles of $g(u, s, \tau, \lambda)$ at $\tau_{m}=\tau_{m}(\lambda)$ and residues $-X_{m}(u, \lambda) X_{m}(s, \lambda)$. These "eigenfunctions" and "eigenvalues" are not real for nonreal $\lambda$ as can be seen from (5.1) and its conjugate; namely:

$$
\operatorname{Im}\left(\tau_{m}\right) \int_{0}^{\infty}\left|X_{m}(u)\right|^{2} d u+\operatorname{Im}(\lambda) \int_{0}^{\infty} u^{2}\left|X_{m}(u)\right|^{2} d u=0
$$

By analogy with the self-adjoint case, he then speculates that the Green's function of the original problem would take the form

$$
G(u, v, s, t, \lambda)=\sum_{0}^{\infty} X_{m}(u, \lambda) X_{m}(s, \lambda) \gamma\left(u, t,-\tau_{m}, \lambda\right)
$$

and that this is not an expansion which falls within the ordinary theory because of the complex values which appear. To avoid this he introduces the function 


$$
H(u, s, t, \lambda)=\lim _{\nu \rightarrow 0} \int_{0}^{\lambda} \operatorname{Im} G(u, v, s, t, \mu+i \nu) d \mu
$$

which involves real value of $\lambda$ only and shows that $H$ is of bounded variation in any finite $\lambda$-interval and furthermore that it is the integral over $(0, \lambda)$ of the function

$$
h(u, v, s, t, \lambda)=\sum_{0}^{\infty} X_{m}(u, \lambda) X_{m}(s, \lambda) \operatorname{Im} \gamma\left(v, t,-\tau_{m}, \lambda\right)
$$

where this last series is absolutely convergent for $v \neq t$. From this he deduces that the spectrum of the original problem is continuous from $-\infty<\lambda<\infty$ and proves an expansion theory for an arbitrary function of the original problem.

While the connection of his procedure to that of the theory of dissipative operators will be commented upon in the next section, it is clear that it furnishes further evidence of our main point that nonself-adjoint problems arise naturally and that it is often a matter of choice whether to circumvent them or not.

Finally, in view of the problem involving the optical model of the nucleus (complex potential) and wave propagation problem through non-isotropic media, it would be highly desirable to have a generalization of the theory of Titchmarsh's second volume (XV, Part II). A start in this direction has been made by the Russian school beginning with Gelfand [31] who established the following:

THEOREM 5.3. Let the operator $L(u)=-\nabla^{2} u+p u$ for a real-valued function $p$ be self-adjoint in $L_{2}$ and let $q$ be a complex valued function vanishing outside a set of compact support, then: (a) the discrete complex spectrum of $L(u)+q u$ is denumerable and possesses only real limit points and; (b) the continuous spectrum of $L(u)$ and $L(u)+q u$ on the real axis are the same.

According to Naimark [78], these results have been generalized by R. Martirosjin in a Moscow dissertation (1954) to whom he attributes the following generalization of a theorem of his (cf. [77]) for ordinary differential equations.

THEOREM 5.4. Consider the operator $-\nabla^{2} u+q u$ with a complex function $q$ and let $r$ denote the distance in $E_{n}$. Then if for $\epsilon>0, q e^{+e r}$ is bounded and integrable in $E_{n}$, the discrete spectrum for $n=2$ has zero as its only limit point and for $n=3$ the discrete spectrum consists of $a$ finite number of points.

Clearly our knowledge of this type of operator is as yet very 
meager and its systematic discussion remains an open problem. ${ }^{10}$

6. The theory of the non-self-adjoint operators of Livsic. In an attempt to generalize Schur's theorem on the triangularization of finite matrices to a class of non-self-adjoint operators, Livsǐc [67] introduced the operators of class $(i \Omega)$ with the properties

(1) The region of definition of an operator $A$ of class $(i \Omega)$ is dense in a Hilbert space. Hence the adjoint operator $A^{*}$ exists.

(2) An operator $A$ of class $(i \Omega)$ is closed. Therefore $A^{* *}$ exists and is equal to $A$.

(3) The "imaginary part" of $A, S=\left(A-A^{*}\right) /(2 i)$ is a completely continuous operator with a finite absolute trace.

(4) The "real part" of $A, R=\left(A+A^{*}\right) / 2$ is bounded.

Unfortunately, we will not be able to comment constructively on all of his results since his methods of proof were based on the use of infinite matrices and remain obscure to us and to all other American mathematicians known to us who have attempted to follow them. ${ }^{11}$ On the other hand, certain of his results have been obtained by operator methods and some even without the use of his assumption (4). These in particular can be found in Dolph and Penzlin [24] and they are as follows:

THEOREM 6.1. Every point outside the real $\lambda$-axis is either a regular point or an eigenvalue of finite multiplicity.

TheOREM 6.2. Let $\sigma_{1} \leqq(S \psi, \psi) /(\psi, \psi) \leqq \sigma_{2}$.

Then the points $\operatorname{Im} \lambda>\sigma_{2}>0$ and $\operatorname{Im} \lambda<\sigma_{1} \leqq 0$ are regular points of the operator $A$ of class (i $\Omega$ ).

${ }^{10}$ A review of R. Martirosjin's paper entitled, On the spectrum of the non-selfadjoint operator $-\nabla^{2} u+c u$ in three dimensional space, has just appeared, (MR 2106, vol. 21 no. 4 (1960) pp. 403-404). His results were published in Izv. Akad. Nauk Armyan SSR Ser. Fiz. Mat. Nauk vol. 10 no. 1 (1957) pp. 85-111 (Russian) and in addition to the result stated above, he has also proved: (1) The entire positive real axis belongs to the spectrum of the operator $T$ of the title; (2) Any other points of the spectrum of this operator are eigenvalues; (3) The resolvent of the operator $T$ is an integral operator with a kernel $H(P, Q, \lambda)$ which has the property that, considered as a function of either $P$ or $Q$ separately, " ||$H \|_{2}$ is bounded uniformly in the other variable by a bound depending on $\lambda$ only; (4) If $\lambda=\xi+i \eta$ and if $\alpha=\|c\|^{2} / 8 \pi$ then the entire spectrum of $T$ lies inside the parabola $\eta^{2}=\alpha^{2}\left(\xi+\alpha^{2}\right)$ "; (5) "If $c$ is summable (as well as square-summable and bounded), the nonpositive point spectrum is bounded."

"For an earlier summary of the work of Livsic which gives some indication of his Volterra spectral operator, see Naimark (77). A more readable account of a special case of the general theory can be found in M. Brodskii and M. Livsic, Spectral analysis of non-self-adjoint operators and intermediate systems, Trans. Amer. Math. Soc. vol. 13 (1960) pp. 265-346. 
TheOREM 6.3. The eigenvalues $\left\{\lambda_{n}\right\}$ of $A$ satisfy the inequality

$$
\sum_{n}\left|\lambda_{n}\right| \leqq \sum_{m}\left|\sigma_{m}\right|
$$

Here $\sigma_{m}$ are the eigenvalues of the "imaginary part" of $A, S$ and both $\lambda_{n}$ and $\sigma_{m}$ are to be taken according to their multiplicity.

TheOREM 6.4. The number of eigenvalues $\lambda_{p}$ of $A$ with $\operatorname{Im} \lambda_{p} \neq 0$ is at most denumerable. Zero is the only possible limit point of $\operatorname{Im} \lambda_{p}$.

7. Non-self-adjoint dissipative operators. For the physically more interesting sub-class of "dissipative operators," a term first introduced by Mukminov, [75] many more properties have been established by operator methods. Depending upon the use to which this concept is intended, we have the definition as follows:

Definition. Let $\mathcal{H}$ be a Hilbert space with an inner product $\langle y, z\rangle$. A linear operator $A$ with domain $D(A)$ is said to be dissipative if either

$$
\operatorname{Im}\langle A y, z\rangle=\frac{1}{2 i}\{\langle A y, y\rangle-\langle y, A y\rangle\} \geqq 0 \quad(\leqq 0),
$$

or

$$
\operatorname{Re}\langle A y, y\rangle=\frac{1}{2}\{\langle A y, y\rangle+\langle y, A y\rangle\} \leqq 0 \quad(\geqq 0)
$$

The operator $A$ is said to be maximal dissipative if it is not the proper restriction of any other dissipative operator.

The physical interpretation of this concept differs in detail depending upon the physical application. For example, in scattering problems it is the requirement that harmonic wave forms do not grow in time in a lossless medium. In reality all media are somewhat lossy and it is customary to allow the wave number to have a small imaginary part so that some attenuation will result. For this type of problem, examples of dissipative operators are furnished by the free-space Green functions for the one and three-dimensional Helmholtz equation. Thus for functions $\phi$ vanishing outside sets of compact support we have from Dolph and Ritt [21], for one dimension, the relation

$$
\begin{aligned}
& \operatorname{Im}\left\{\int_{-\infty}^{\infty} \int_{-\infty}^{\infty} \phi(x) \frac{e^{i k|x-y|}}{2 i k} \phi^{*}(y) d x d y\right\}^{\prime} \\
& =-\frac{1}{4 k}\left\{\left|\int_{-\infty}^{\infty} e^{-i k x} \phi(x) d x\right|^{2}+\left|\int_{-\infty}^{\infty} e^{i k x} \phi(x) d x\right|^{2}\right\} \leqq 0
\end{aligned}
$$


and from Dolph [19] for three dimensions the relation

$\operatorname{Im} \iint \phi(r) \frac{e^{i k\left|r-r^{\prime}\right|}}{4 \pi\left|r-r^{\prime}\right|} \phi^{*}\left(\boldsymbol{r}^{\prime}\right) d^{3} r d^{3} r^{\prime}=\frac{k}{8 \pi} \int d \Omega\left|\int e^{-i k \cdot \boldsymbol{r}^{\prime}} \phi\left(\boldsymbol{r}^{\prime}\right)^{3}\right|^{2} \geqq 0$.

While these are stated for real $k$, they may be extended to $\operatorname{Im} k>0$. The fact that these operators are dissipative can be given a direct physical interpretation with the aid of the cross-section or optical theorem which states that the imaginary part of the forward scattering amplitude is proportional to the total cross-section of the scattering process. In particular for the quantum mechanical case, it is not difficult to establish that

$$
\frac{8 \pi}{k} \operatorname{Im} \iint V(\boldsymbol{r}) \psi(\boldsymbol{r}) \frac{e^{i k\left|\boldsymbol{r}-\boldsymbol{r}^{\prime}\right|}}{4 \pi\left|\boldsymbol{r}-\boldsymbol{r}^{\prime}\right|} V\left(\boldsymbol{r}^{\prime}\right) \psi^{*}\left(\boldsymbol{r}^{\prime}\right) d^{3} r d^{3} \boldsymbol{r}^{\prime}=\sigma=\frac{4 \pi}{k} \operatorname{Im} f(0, \psi)
$$

for all potential such that the indicated integrals exist. Here $f(0, \psi)$ denotes the forward scattering amplitude, $\sigma$ the total cross-section. (For details see Dolph and Penzlin [24].)

In his work on the abstract theory of hyperbolic systems of partial differential equations, Phillips [84], notes that the condition that the spatial part of the system be dissipative is equivalent to the fact that the energy of the system is nonincreasing in time. Interpretations similar to these are possible in all other cases known to the author. ${ }^{12}$ Mathematically, as we shall see, the dissipative condition, seems to be the natural extension of the notion of positive-definiteness for selfadjoint operators and in particular it is sufficient to guarantee the existence of a resolvent operator in a half-plane in many instances.

The first result on dissipative operators obtained by Hilbert space methods is the theorem of Livsic-Mukminov [67; 75] which is as follows:

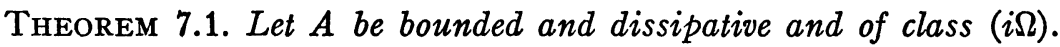
Let $\mathcal{E}=$ the range of $S$, and let $\mathcal{G}$ be the largest subspace of $\mathcal{E}^{\perp}$ invariant under $A$. Then if $\mathcal{G}$ is the null space, the principal functions of $A$ are complete in the range of $A$ if and only if

$$
\operatorname{Im} \sum \lambda_{k}=\sum \sigma_{k}=\text { Trace } S \text {. }
$$

Since there are several obscure points in the proof by Mukminov, ${ }^{13}$

12 Compare, for example, H. König and J. Meixner, Lineare Systeme und lineare Transformationen, Math. Nachr. vol. 19 (1958) pp. 265-322, for a recent account of the role of dissipative operators in passive network theory and in linear thermodynamical systems.

18 The author is indebted to R. S. Phillips for his help in clarifying Mukminov's proof. 
we will elaborate here. The proof proceeds as the proof of theorem (6.3) until the inequality is established for $\left|\sigma_{k}\right|=\sigma_{k}>0$. Let us next suppose that the orthonormal set of principal vectors of $A$, the set $\left\{\phi_{p}\right\}$, is complete in the range of $A$. We observe that the range of $A$ contains the range of $S$ for if $f$ were an element orthogonal to the range of $A$, we would have $(A f, f)=(R f, f)-i(S f, f)=0$ which in turn implies $(S f, f)=0$ which is impossible unless $f=0$ because of the dissipative nature of $S$. Now the inequality of Theorem $6.1 \mathrm{can}$ in this case be written as

$$
\sum \operatorname{Im} \lambda p=\sum_{q} \sigma_{q} \sum_{p}\left|\left\langle\phi_{p}, \omega_{q}\right\rangle\right|^{2} \leqq \sum \sigma_{k}
$$

since all the $\sigma_{k}$ are positive. Here $S \omega_{q}=\sigma_{q} \omega_{q}$. Parseval's quality

$$
\sum_{p}\left|\left\langle\phi_{p}, \omega_{q}\right\rangle^{2}\right| \leqq\left\|\omega_{q}\right\|^{2}=1
$$

now implies that the equality holds in (5.1). Conversely, if the quality sign holds in (7.2), hence in (7.3), we see that the set $\left\{\omega_{a}\right\}$ lies in the space spanned by $\left\{\phi_{p}\right\}$, hence in the space spanned by the nonorthogonalized principal functions say $\left\{\psi_{p}^{\alpha}\right\}$. Thus any function orthogonal to all the $\left\{\psi_{p}^{\alpha}\right\}$ is also orthogonal to the set $\left\{\omega_{q}\right\}$. Now let $\mathcal{G}^{*}$ consist of all the functions in the range of $A$ orthogonal to the set of principal functions $\left\{\psi_{p}^{\alpha}\right\}$. Then for $g \in \mathcal{G}^{*}$, we have $0=\left(g, \omega_{q}\right)$ $=\left(g,\left(S / \sigma_{q}\right) \omega_{q}\right)=\left(1 / \sigma_{q}\right)\left(S g, \omega_{q}\right)$ so that $g$ is also in the space $\mathcal{E}^{\perp}$. Thus we have

$$
\left\langle A g, \psi_{p}^{\alpha}\right\rangle=\left\langle A^{*} g, \psi_{p}^{\alpha}\right\rangle=\left\langle g, A \psi_{p}^{\alpha}\right\rangle=\left\langle g, \lambda_{p} \psi_{p}^{\alpha}+\psi_{p-1}^{\alpha}\right\rangle=0 .
$$

Since $A$ is self-adjoint in $\varepsilon^{\perp}$ thus the set $\mathrm{g}^{*}$ is invariant under $A$. However since $\mathcal{G}^{*}$ is also contained in $\mathcal{E}^{\perp}$, it must be contained in $\mathcal{G}$, the largest invariant set in $\mathcal{E}^{\perp}$, and this was assumed to be the null set. Mukminov [75] also announced the following theorem:

Theorem 7.2. If $A$ is dissipative, bounded and of class (is) and if

$$
\sum_{J_{j} k=1}^{\infty} \frac{\operatorname{Im} \lambda_{j} \operatorname{Im} \lambda_{k}}{\left|\lambda_{j}-\lambda_{k}\right|^{2}}<\infty,
$$

then for any element $f$ in $\mathfrak{H C}$ of the form

the following inequality

$$
f=\sum_{k=1}^{n} c_{k} \phi_{k}
$$




$$
m \sum_{k=1}^{n}\left|c_{k}\right|^{2} \leqq\|f\|^{2} \leqq M \sum_{k=1}^{n}\left|c_{k}\right|^{2}
$$

holds where $M$ and $m$ are positive constants that do not depend upon $f$.

A generalization of this theorem has recently been announced and a proof sketched by Glasmann [34] under the weaker hypothesis that $A$ is merely a bounded, dissipative operator with an infinite system of orthonormalized eigenfunctions. ${ }^{14}$ For these operators there is also the useful theorem of Dolph and Ritt [21]:

TheOREM 7.3. Let $A=R-i S$ be dissipative and of class i $\Omega$, and let $\eta$ be the intersection of the null spaces of the operators $I-R$ and $S$. Then $\eta$ is the null space of $T=I-A$ and the space $\mathfrak{H}_{1}=\eta^{\perp}$ remains invariant under $A$. $\eta$ is also the null space of $T^{*}=I-A^{*}$ and $\mathfrak{H}_{1}$ remains invariant under $A^{*}$. Further $(T \phi, \psi)=\left(T \phi_{1}, \psi_{1}\right)$ where $\phi, \psi$ are arbitrary elements in the Hilbert space $\mathfrak{H C}$ and where $\phi_{1}, \psi_{1}$, are their respective orthogonal projections on $\mathfrak{F C}_{1}$.

Two independent approaches to the problem of unbounded dissipative operators have been taken by Glasmann [33] and Dolph and Penzlin [24]. Glasmann defined a nonlinear operator $J$ on the entire Hilbert space with the properties that

$$
\begin{gathered}
(J f, J g)=(f, g), \\
J^{2} f=f,
\end{gathered}
$$

and called an operator $J$-symmetric if

$$
(A f, J g)=(f, J A g) \text {, }
$$

for $f, g$ in the domain of $A$. If the closure of the domain of $A$ is the entire Hilbert space, then the adjoint operator $A^{*}$ exists and

$$
J A J \subset A^{*} \text {. }
$$

An operator is then called $J$-self-adjoint if $J A J=A^{*}$. His main result was the theorem:

Theorem 7.4. A J-symmetric dissipative operator $A$ with a dense domain can be extended to $A$, which is J-self-adjoint.

Both Glasmann and Dolph and Penzlin obtained the existence of $(A-\lambda I)^{-1}$ in a half plane and the estimate

${ }^{14}$ Further results on dissipative operators obtained without the use of infinite matrices have been announced by M. Krein, A contribution to the theory of linear non-self-adjoint operators, Soviet Math. Amer. Math. Soc. vol. 1 no. 1 (1960) pp. 38-40. 


$$
\left\|(A-\lambda I)^{-1}\right\| \leqq \frac{1}{\operatorname{Im} \lambda}, \quad \operatorname{Im} \lambda>0,
$$

for dissipative operators and Glasmann introduced the notion of deficiency numbers which are defined as the dimension $m$ of the orthogonal complement of the space $(A-\lambda I) D_{A}$ where $D_{A}$ is the domain of $A$. He demonstrated that $m$ was a constant for $\operatorname{Im} \lambda \geqq 0$, and that an operator was $J$-self-adjoint if and only if $m=0$.

While Glasmann utilized the methods of Krein [51] and an appropriately defined Cayley transform to obtain his results, Dolph and Penzlin [24] approached the problem from the view-point of the Riesz approach to the spectral theorem as it is found in Achieser and Glasmann [I] and noted that

$$
\left\langle(A-\lambda I)^{-1} \phi, \phi\right\rangle
$$

was an analytic function with positive imaginary part in the half plane $\operatorname{Im} \lambda>0$ with enough additional properties to enable it to be represented as

$$
\left\langle(A-\lambda I)^{-1} \phi, \phi\right\rangle=\int_{\infty}^{\infty} \frac{d_{t} \omega(t, \phi)}{t-\lambda}
$$

where $\omega(t, \phi)$ is a uniquely determined monotonic function normalized by the conditions $\omega(-\infty, \phi)=0, \omega(+\infty, \phi)=\|\phi\|^{2}$. For this representation they assumed explicitly that

(1) $A=R-i S$, where $R$ and $S$ are Hermitian operators;

(2) The operator $S$ is positive semi-definite $\operatorname{Im} \lambda>0$;

(3) The range of the operator $(A-\lambda I)$ is the entire Hilbert space for $\operatorname{Im} \lambda>0$.

Under these hypotheses it follows then that

THEOREM 7.5. Under (1), (2), and (3), the resolvent operator $(A-\lambda I)^{-1}$ exists in the complex half-plane $\operatorname{Im} \lambda>0$ as a bounded operator and has a weak representation in the form

$$
\left\langle(A-\lambda I)^{-1} \phi, \psi\right\rangle=\int_{\infty}^{\infty} \frac{\langle F(t) \phi, \psi\rangle}{t-\lambda} ; \operatorname{Im} \lambda>0,
$$

where $F(\lambda)$ is a uniquely determined self-adjoint operator with the properties

(a) $F(\lambda)$ is positive-definite and bounded $0 \leqq F(\lambda) \leqq I$;

(b) The family $F(\lambda)$ is nondecreasing for $F(\lambda) \leqq F\left(\lambda^{\prime}\right), \lambda<\lambda^{\prime}$.

It should be noted that the second hypothesis is automatically fulfilled if $S=0$ while the third will hold if $R$ is a maximal Hermitian 
(not necessarily self-adjoint) operator or if $R$ and $S$ are self-adjoint and $S$ is bounded. Also the author is indebted to E. A. Coddington for the observation that the representation given above is unique in spite of the statement to the contrary in [24]. The uniqueness follows from the fact that the above representation implies that

$$
\left(A^{*}-\lambda I\right)^{-1}=\int_{-\infty}^{\infty} \frac{d F(t)}{t-\lambda} \quad \text { for } \operatorname{Im} \lambda<0
$$

so that any non-uniqueness in $F$ would imply a non-uniqueness in $A^{*}$ in contradiction to its known uniqueness. ${ }^{15}$ Now the family $F(\lambda)$ is a generalized resolution of the identity in the sense of Naimark and thus by his theorem (Cf. Appendix I of [I]), there exists a larger Hilbert space $\mathfrak{H}^{+}$containing the given Hilbert space $\mathfrak{H}$ as a subspace and a corresponding orthogonal resolution of the identity $E(\lambda)$ in $\mathcal{F}^{+}$such that for each element $\phi$ in $\mathfrak{T C}$ one has $F(\lambda) \phi=P^{+} E(\lambda) \phi$ where $P^{+}$is the orthogonal projection operator of $\mathfrak{F C}^{+}$into $\mathfrak{F}$. It is also easy to see that the self-adjoint operator $A^{+}$in $\mathfrak{F}^{+}$associated with $E(\lambda)$ has the property that $P^{+}\left(A^{+}-\lambda I\right)^{-1}=(A-\lambda I)^{-1}$ for if $\phi, \psi$ are any two elements of $\mathfrak{K}$, then

$$
\begin{aligned}
\left(P^{+}\left(A^{+}-\lambda I\right)^{-1} \phi, \psi\right) & =\left(\left(A^{+}-\lambda I\right)^{-1} \phi, P^{+} \psi\right)=\int_{-\infty}^{\infty} \frac{d\left(E(t) \phi, P^{+} \psi\right)}{t-\lambda} \\
& =\int_{-\infty}^{\infty} \frac{d(F(t) \phi, \psi)}{t-\lambda}=\left((A-\lambda I)^{-1} \phi, \psi\right) .
\end{aligned}
$$

Questions concerning the detailed structure of $A^{+}$are still open although in the case where it arises from symmetric ordinary differential equations many properties of its resolvent have been determined. ${ }^{15}$

Since the original write-up did not adequately discuss the selfadjointness of $F(\lambda)$, I would like to take this opportunity to amplify this point. It follows from a known form of the Stieltjes inversion theorem (cf. Greenstein [35]) that (5.3) can be inverted to yield

$$
\omega\left(t_{2}, \phi\right)-\omega\left(t_{1}, \phi\right)=\lim _{\nu \rightarrow 0^{+}} \frac{1}{\pi} \int_{t_{1}}^{t_{2}} \operatorname{Im}\left\langle(A-\lambda I)^{-1} \phi, \phi\right\rangle d x .
$$

Letting $\psi_{\lambda}=(A-\lambda I)^{-1} \phi$ so that $\phi=(A-\lambda I) \psi_{\lambda}$ for $\operatorname{Im} \lambda>0, \lambda=x+i y$ this can be written as

${ }^{15}$ Cf. E. A. Coddington, Generalized resolutions of the identity for symmetric ordinary differential equations, Ann. of Math. vol. 68 (1958) pp. 378-392 and E. A. Coddington and R. C. Gilbert, Generalized resolvents of ordinary differential equations, Trans. Amer. Math. Soc. vol. 93 (1939) pp. 216-241. 


$$
\begin{aligned}
\omega\left(t_{2}, \phi\right)-\omega\left(t_{1}, \phi\right) & =\lim _{\nu \rightarrow 0^{+}} \int_{t_{1}}^{t_{2}} \operatorname{Im}\left\langle\psi_{\lambda},(A-\lambda I) \psi_{\lambda}\right\rangle d x \\
& =\lim _{\nu \rightarrow 0^{+}} \int_{t_{1}}^{t_{2}} \operatorname{Im}\left\langle\psi_{\lambda},(S+y) \psi_{\lambda}\right\rangle d x \\
& =\lim _{\nu \rightarrow 0^{+}} \int_{t_{1}}^{t_{2}}\left\langle R_{\lambda} \phi,(S+y) R_{\lambda} \phi\right\rangle d x
\end{aligned}
$$

Now if $t_{1}$ is allowed to approach $-\infty$ (there is no difficulty here since the integrand is positive) from the normalization we obtain

$$
\omega\left(t_{2}, \phi\right)=\lim _{y \rightarrow 0^{+}} \int_{-\infty}^{t}\left\langle R_{\lambda} \phi,(S+y) R_{\lambda} \phi\right\rangle d x .
$$

This may now be polarized by means of the definition

$$
\omega(t, \phi, \psi)=\omega(t, f+g)-\omega(t, f-g)+i \omega(t, f+i g)-i \omega(t, f-i g)
$$

and the new functional is readily seen to be bilinear and it is easily proven bounded as in Achieser-Glasmann [1] so that one can apply the Riesz representation theorem to it to deduce the existence of an operator $F(\lambda)$ such that

$$
\omega(t, \phi, \psi)=\langle F(t) \phi, \psi\rangle .
$$

However $0 \leqq \omega(t, \phi, \phi)=\langle F(t) \phi, \phi\rangle$ so that it follows from a known theorem that $F(\lambda)$ must be positive and self-adjoint. From this representation theorem one can deduce expansion theorems as in the selfadjoint case by integrating the left-hand side around a suitably chosen contour in the complex $\lambda$-plane when one possesses sufficiently detailed knowledge of the singularities of the integrand. In view of the similarity between (5.5) and (7.5), it is apparent that Titchmarsh's formula (5.5) can now be subsumed as part of the general theory of dissipative operators.

Dissipative operators occur in many applications and even naturally in mathematical theory as we have observed in the case of Titchmarsh's treatment. Since the resolvent exists in half of the complex plane, it naturally follows that all, if any, complex eigenvalues must exist in the other half. While the existence of a relation of the form (5.4) which confines the eigenvalue to a half-plane does not logically imply that the associated operator is dissipative, experience seems to indicate that in practice it does. In particular, the operators considered by Sims [92] for which such a relation is easily demonstrated (cf. Dolph [18]) are dissipative and we have, more generally, the following theorem by Glasmann [33]. 
Theorem 7.6. Let $L(y)=\sum_{j}^{n}(-1)^{k}\left(d^{k} / d x^{R}\right)\left[P_{n-k}(x)\right]\left(d^{k} y / d x k\right)$ be the formal differential operator acting in $L_{2}(0, \infty)$ on functions $y=\phi(x)$ which, together with their first $(2 n-1)$ derivatives are absolutely continuous and zero at the origin and such that $\operatorname{Im} P_{k}(x) \geqq 0$. Then (1) the number of linearly independent solutions of $L(y)=\lambda y, \operatorname{Im} \lambda>0$, does not depend upon $\lambda$, and is always greater than or equal to $n$. (2) The operator $L(y)$ with minimal domain of definition can be extended to a $J$-self-adjoint dissipative differential operator. (3) The resolvent can be written in the form

$$
R_{\lambda} y=[L-\lambda I]^{-1} y=\int_{0}^{\infty} G(x, s, \lambda) y(s) d s,
$$

where the kernel is real symmetric $G(x, s, \lambda)=G(s, x, \lambda)$ and if the deficiency index $m<2 n$, then the integral

$$
\int_{0}^{\infty}|G(x, s, \lambda)|^{2} d s<\infty,
$$

while if the deficiency index $m=2 n$, the double integral

$$
\int_{0}^{\infty} \int_{0}^{\infty}|G(x, s, \lambda)|^{2} d x d s<\infty
$$

and the resolvent is a compact operator.

The concept of a dissipative operator has been used by Phillips [84] to develop a general theory of Cauchy problem for a system of hyperbolic partial differential equations, from the point of view of semi-groups. In particular, he considers the initial value problem $y_{t}=L y=E^{-1}\left\{\left(A^{i} y\right)+B y\right\}$

$$
y(x, 0)=f(x)
$$

for functions $\{y\}$ of $x, t$ with values in a $k$-dimensional complex euclidean space when a domain $\Delta$ is given in a $m$-dimensional real euclidean space with points $x=x_{1}, \cdots, x_{m}$. Tensor notation is used for summation and the index $i$ denotes differentiation with respect to $x_{i} . E, A^{i}$ and $B$ represent $k \times k$ matrix-valued functions of $x$ alone, $E$ is positive-definite, $A^{i}$ is hermitian and the dissipative condition

$$
B+B^{*}+A_{i}^{i} \leqq 0 \quad \text { for } x \in \Delta
$$

holds where $B^{*}$ is the adjoint of $B$ relative to the usual hermitian inner product. It is further assumed that in each compact subset of $\Delta$ the elements of $E$ are continuous, the elements of $A^{i}$ are absolutely 
continuous with respect to $x_{i}$, and that the elements of $A_{i}^{i}, B$ are square integrable. Letting $D=E^{-1}\left[B+B^{*}+A_{i}^{i}\right]$ and noting that if $y(x, t)$ is a solution of the system (7.5), the energy of the associated physical model is given by

$$
\frac{1}{2} \int_{\Delta}(E y, y) d x .
$$

In terms of a Hilbert space $\mathfrak{H C}_{0}$ with inner product defined by

$$
\langle y, z\rangle=\int_{\Delta}(E y, z) d x
$$

the rate of change of energy from (7.6) is given by

$$
\langle y, y\rangle_{t}=\left\langle L_{1} y, y\right\rangle+\left\langle y, L_{1} y\right\rangle=\int_{\Delta}\langle E D y, y\rangle d x+\int_{\Gamma}\left(A^{i} y, y\right) \eta^{i},
$$

where $n=n_{1}, \cdots, n_{m}$ is the outward normal to the boundary $\Gamma$ of assuming that $n$ exists. Since the condition $\langle y, y\rangle_{t} \leqq 0$ is imposed for all smooth functions vanishing outside compact subsets of $\Omega$, the condition (7.7) follows and has the physical significance that there are not internal energy sources. Solutions to the system (7.6) whose boundary values satisfy

$$
\left(A^{i} y, y\right) n^{i} \leqq 0
$$

will be clearly dissipative. (When the boundary integral is not well defined the last two equalities in (7.8) can be used as a definition). If one now defines the operator $L_{1}$ to be the operator of largest domain of the type (7.6) for which the boundary integral is meaningful in the above extended sense and if $L_{0}$ is the largest restriction of $L_{1}$ and has for its domain only those functions which are essentially zero on the boundary, then the domain of an operator $L$ such that $L_{0}<L<L_{1}$ will be determined entirely by the boundary values assumed by its member functions. Phillips proceeds to characterize all solutions between $L_{0}$ and $L_{1}$ whose boundary values satisfy (7.9). These he calls properly dissipative and there is an equivalence between the class of generators of properly dissipative solutions and maximal dissipative operators between $L_{0}$ and $L_{1}$. In particular, he proves the following:

Theorem 7.7. An operator $L$ is the infinitesimal generator of a strongly continuous semi-group of contraction operators of $\mathrm{FC}_{0}$ if and only if $L$ is a maximal dissipative operator with dense domain. 
As in Glasmann the theory depends upon the use of an appropriate Neumann-Cayley transform and contains many results similar to the usual operator theory such as the following example:

THEOREM 7.8. (a) If $L$ is maximal dissipative and closed, then it has a dense domain. (b) If $L$ is a maximal dissipative operator with dense domain then so is $L^{*}$.

In determining the maximal dissipative generators for this problem there are dual procedures available in that one can begin with a minimal operator $L_{0}$ or a maximal operator $L_{1}$ each of which represents the spatial part of the hyperbolic operator and then seek maximal dissipative extensions of $L_{0}$ and restrictions of $L_{1}$. The first procedure can lead to operators which are no longer merely differential operators whereas the second can result in differential operators whose domains need not be restricted by simple boundary conditions but by "global" lateral conditions. A similar phenomenon occurs in the contractions considered by Sims [92] and in Feller's [25] treatment of parabolic differential equations. The maximal dissipative operators which are differential operators and whose domains are delimited by ordinary boundary conditions are those which are simultaneously extensions of $L_{0}$ and restrictions of $L_{1}$.

The concept of a dissipative operator is useful in still other situations. Thus, as noted earlier, it enabled Müller to eliminate nonelementary divisors from Weyl's diffraction theory. It has been used by Dolph and Ritt [21] and Dolph [19] to show that the critical points of the Schwinger variational principles of scattering theory are saddle points which, since they are unknown $a$ priori, make the construction of a systematic approximation theory difficult if not impossible. Because of the known equivalence of these principles to those of Hulthén-Kohn (cf. Hulthén [40], Kohn [48; 49], Kato[45]) a similar situation exists for all of the widely used variational principles. The concept has also been used by Krein and Gohberg [54] in their review article on root spaces where they consider perturbations of selfadjoint operators in Hilbert space in particular and obtain results similar to those of Theorem (6.3). Finally, it should be noted that Lidskii [64] has treated non-self-adjoint operators possessing discrete spectra in root spaces.

In view of the rapid progress to date and the intimate relationship between physical theory and dissipative operators it seems safe to conjecture that the time is not far distant when the theory will be considered a standard part of an advanced mathematical education. 
8. Initial value problems and singular integral equation techniques. It is well known that many problems when formulated as singular integral equations with a Cauchy kernel have led to closed form solutions (cf. Muskhelishvili [XII] and Tricomi [XVI]. What perhaps is not so well known or appreciated is the fact that these techniques lie behind much of the recent work on dispersion relations in transport phenomena, quantum potential scattering, and quantum field theory scattering.

A unified approach to these problems has been developed by Case in a series of papers and lectures in which he has expanded upon the ideas initiated by Van Kampen $[98 ; 99]$. The approach has been two fold: on the one hand, it involves the systematic use of Laplace and Fourier transform theory; and on the other, it involves the construction of a theory of elementary solutions which in general are distributions and not functions but which nevertheless with the aid of singular integral equation theory are shown to satisfy completeness relations in an $L_{1}$ type of theory reminiscent of the more usual $L_{2}$ theory. Furthermore the complete equivalence of the two approaches can be demonstrated. Specifically the achievements to date of this theory are as follows:

(1) In [11], Case treats the one-dimensional neutron transport equation with the aid of this distribution theory and demonstrates that it is sufficient to consider that part of the continuous spectrum which is confined to the real axis.

(2) In [10], Case treats the plasma oscillation problem associated with the name of Landau [57], and demonstrates the complete equivalence of Landau's original approach to the initial value problem via the Laplace transform with that of Van Kampen's [97].

(3) In $[12 ; 13 ; 14 ; 15]$, he applies this theory to a series of linearized flow problems. In particular in [12] he determines distribution solutions to the inviscid linearized Navier-Stokes equations which are limits of the linearized viscous equations as the viscosity goes to zero even though there are no such solutions in terms of ordinary functions thus completing the analogy that exists between quantum and classical mechanics in the limit as Planck constant goes to zero.

(4) In his lectures [16] he has approached quantum scattering theory for a class of problems in which the kernel of the relevant Fredholm integral equation can be approximated in $L_{2}$ by degenerate kernels composed of a finite sum of products of orthonormal functions. For any finite approximation, the scattering problem can be solved in closed form by the methods of the Landau theory and the asymptotic relations of scattering theory derived explicitly. Further- 
more, double dispersion relations of the type conjectured by Mandelstam [72] for the quantum field case can be rigorously established.

Throughout this entire theory, little more than the Plemelj formulas are used in connection with the observation that the relevant physical quantities are boundary values of functions analytic in a suitably cut complex plane. As such this approach seems closest to that now being attempted in the discussion of dispersion relations for quantum field theory where deep theorems on the nature and continuability of functions of several complex variables are needed as can be seen by reference to the works of Wightman [104], Wightman, Hall [105], and to the book by Bogoliubov and Shirkov [III]. Finally, this same approach can be used to describe Wiener-Hopf integral equation theory in terms of singular integral equations so that, pedagogically at least, unity is achieved for the problems treated by this method. Of course this equivalence which Case and the author have shown by three different methods makes it immediately clear that a common line of analyticity is sufficient for the Wiener-Hopf method but this is a result first obtained by Sparenberg $[93 ; 94]$ by a somewhat different chain of reasoning.

In view of this and the impact these methods will undoubtedly have on future developments, a brief sketch of some of the underlying ideas will be attempted in what is perhaps the simplest case, that of longitudinal plasma oscillations. While the word plasma denotes any jelly-like substances, the plasmas of physics are usually gases which contain a suitably high density of free positive and negative electrically-charged particles and a plasma is regarded as a medium which tends to remain near its free field state and neutral equilibrium state because of the long range Coulomb forces which are present in it. In many ways a plasma is similar in behavior to a liquid which tends to remain near its equilibrium state of a definite volume and which resists efforts to produce a change in this volume. However, in this process of resisting change, there is built in a oscillatory process in that when a given volume contains an excess of molecules, the resulting pressure gradient will create a net flux out of the region which will continue on beyond the state of uniform density until the momentum can be reversed. The resulting process produces sound waves which the liquid can transmit. In plasmas similar phenomena take place because of the presence of the electro-magnetic forces and as a result they have often been termed the fourth state of matter. An excellent introduction to their behavior can be found in the papers by Bohm and Gross [3]. Here for simplicity we shall restrict ourselves to the case where the positive ions are to be thought of as uniformly spread 
over the region under consideration (because of their greater mass, they are much less able to move when subjected to small forces) and further we shall limit consideration to one Fourier space component of the system. In this case, the phenomena of small (i.e. linearized) longitudinal oscillation can be reduced to consideration of the differential-integral equation

$$
\frac{\partial y}{\partial t}+i k y(v)=-i k n(v) \int_{-\infty}^{\infty} y\left(v^{\prime}\right) d v^{\prime}
$$

for the unknown distribution function of longitudinal velocity. In this equation $\eta(v)$ is a known function of logitudinal velocity and $k$ is the magnitude of the transform parameter. (For a detailed derivation, see Van Kampen [98] or Case [10].) An equation adjoint to (8.1) is defined by

$$
\frac{\partial z}{\partial t}+i k z=-i k \int_{-\infty}^{\infty} n\left(v^{\prime}\right) z\left(v^{\prime}\right) d v^{\prime}
$$

and the problem of elementary solutions proportional to $\exp (-i \omega t)$ is considered for each of these. Labelling the solutions with $\mu=\omega / k$ and using the same notation for the time independent part the equations to be considered are

$$
(v-\mu) y_{\mu}(v)=-n(v) \int_{-\infty}^{\infty} y_{\mu}\left(v^{\prime}\right) d v^{\prime},
$$

and

$$
(v-\mu) z_{\mu}(v)=-\int_{-\infty}^{\infty} z_{\mu}\left(v^{\prime}\right) d v^{\prime} .
$$

The solutions of (8.3) can be normalized because of homogeneity so that

$$
\int_{-\infty}^{\infty} y_{n}\left(v^{\prime}\right) d v^{\prime}=1
$$

while those of (8.4) are normalized according to

$$
\int_{-\infty}^{\infty} n\left(v^{\prime}\right) z_{n}\left(v^{\prime}\right) d v^{\prime}=1
$$

The usual argument shows that

$$
\left(\mu-\mu^{\prime}\right) \int_{-\infty}^{\infty} z_{\mu^{\prime}}\left(v^{\prime}\right) y_{\mu}\left(v^{\prime}\right) d v=0,
$$

for all $\mu \neq \mu^{\prime}$. Several cases can now occur. Letting $\delta$ be the Dirac 
delta function and using $P$ to signify that the principal value is to be taken when an expression is integrated with respect to velocity, these are:

I. $\mu$ real and $\eta(\mu) \neq 0$.

The solutions to (8.3) are

$$
y_{\mu}(v)=-P \frac{n(v)}{v-\mu}+\lambda(\mu) \delta(v-\mu)
$$

where

$$
\lambda(\mu)=1+P \int_{-\infty}^{\infty} \frac{n(v) d v}{v-\mu}
$$

while the solutions to (8.4) are

$$
z_{\mu}(v)=-\frac{P}{v-\mu}+\tau(\mu) \delta(v-\mu)
$$

where

$$
\tau(\mu) \eta(\mu)=1+P \int_{-\infty}^{\infty} \frac{n(v) d v}{v-\mu}=\lambda(\mu) .
$$

The normalization condition is

$$
\int_{-\infty}^{\infty} z_{\mu^{\prime}}(v) y_{\mu}(v) d v=\delta\left(\mu-\mu^{\prime}\right) \frac{\lambda^{2}(\mu)+\pi^{2} n^{2}(\mu)}{n(\mu)}=c_{\mu} \delta\left(\mu-\mu^{\prime}\right) .
$$

II. $\mu$ real and $\eta(\mu)=0$.

The solutions to (8.3) are the same as I but principal values are unnecessary.

The solutions to (8.4) are

$$
z_{\mu}(v)=\delta(v-\mu) .
$$

The normalization condition is

$$
\int_{-\infty}^{\infty} z_{\mu^{\prime}}(v) y_{\mu}(v) d v=\lambda(\mu) \delta\left(\mu-\mu^{\prime}\right) .
$$

III. $\mu$ real, $\eta(\mu)=0$ and $\lambda(\mu)=0$. There are only a finite number of such points determined by the respective normalization conditions and for simplicity they are assumed to be simple. The solutions to (8.3) are

$$
y_{i}(v)=y_{\mu_{i}}(v)=-\frac{n(v)}{v-\mu_{i}}, \quad i=1,2, \cdots, m,
$$


while the solutions to (8.4) are

$$
z_{i}(v)=z_{\mu_{i}}(v)=-\frac{1}{v-\mu_{i}} .
$$

The normalization condition is

$$
\int_{\infty}^{\infty} z_{i}(v) y_{i}(v) d v=\delta_{i j}\left\{\int_{\infty}^{\infty} \frac{n(v) d v}{\left(v-\mu_{i}\right)^{2}}\right\}=\delta_{i j} c_{i} .
$$

IV. $\mu$ complex, $\eta(\mu)=0$ and $\lambda(\mu)=0$. Again only a finite number of such points are determined by the respective normalizations and again are assumed simple.

The solutions to (8.3) are

$$
y_{\mu_{i}}(v)=-\frac{n(v)}{v-\mu_{i}},
$$

while the solutions to (8.4) are

$$
z_{\mu_{i}}(v)=-\frac{1}{v-\mu_{i}} .
$$

The normalization condition is an identity.

The above are all easily verified except care must be used for case I, since it requires the Poincaré-Bertrand transformation formula as it is, for example, found in Muskhelishvili [XII]. We now have the following theorem

THEOREM 8.1. The distributions $\left\{y_{\mu}, y_{i}\right\}$ are complete on $-\infty<x<\infty$ for functions satisfying a Hölder condition.

It suffices, for an arbitrary $f(v)$ satisfying a Hölder condition, to consider the solution of the integral equation

$$
f_{1}(v)=f(v)-\sum a_{i} y_{i}(v)=\int A(\mu) y_{\mu}(v) d \mu
$$

for the coefficients $a_{i}$ can be found by the orthogonality relations. Inserting the values of $y_{\mu}(v)$ from (8.5) this integral equation is explicitly

$$
f_{1}(v)=\lambda(v) A(v)+n(v) P \int \frac{A(\mu) d \mu}{u-v},
$$

where 


$$
\lambda(v)=1+P \int_{\infty}^{\infty} \frac{n\left(v^{\prime}\right) d v^{\prime}}{v^{\prime}-v} .
$$

Assuming that $\int A(\mu) d \mu, \int n(v) d v, \int f_{1}(v) d v$ all exist, define the three functions of a complex variable

$$
\begin{aligned}
& N(z)=\frac{1}{2 \pi i} \int \frac{A(\mu)}{\mu-z} d \mu, \\
& Q(z)=\frac{1}{2 \pi i} \int \frac{\eta\left(v^{\prime}\right) d v^{\prime}}{v^{\prime}-z}, \\
& M(z)=\frac{1}{2 \pi i} \int \frac{y\left(v^{\prime}\right) d v^{\prime}}{v^{\prime}-z},
\end{aligned}
$$

and note that these are all analytic with branch cuts along the real axis and that they vanish at infinity like $1 / z$. Letting the superscripts + and - denote the values of these functions as $z$ approaches the real axis from above and below respectively, we have from the Plemelj relations

$$
\begin{aligned}
N^{+}(v)-N^{-}(v) & =A(v), \\
\pi i\left[N^{+}(v)+N^{-}(v)\right] & =P \int \frac{A(v) d v}{\mu-v}, \\
Q^{+}(v)-Q^{-}(v) & =\eta(v), \\
\pi i\left[Q^{+}(v)+Q^{-}(v)\right] & =P \int \frac{\eta(\mu) d \mu}{\mu-v} .
\end{aligned}
$$

These imply

$$
\lambda(v)=1+\pi i\left[Q^{+}+Q^{-}\right]
$$

and

$$
M^{+}(v)-M^{-}(v)=f_{1}(v) .
$$

Insertion into the integral equation yields

$$
\begin{aligned}
\left\{N^{+}(v)\left[1+2 i Q^{+}(v)\right]\right. & \left.-M^{+}(v)\right\} \\
& -\left\{N^{-}(v)\left[1+2 i Q^{-}(v)\right]-M^{-}(v)\right\}=0 .
\end{aligned}
$$

To solve (8.10) we must find a function analytic in the complex plane with cuts along the real axis, which vanishes at infinity, and whose jumps along the real axis satisfy (8.10). If such a function exists the function

$$
J(z)=N(z)[1+2 i Q(z)]=M(z)
$$


has the properties of being analytic in the cut plane, no discontinuities along the cuts, vanishing at infinity and therefore by Liouville's theorem it must be identically zero so that

$$
N(z)=\frac{M(z)}{1+2 \pi i Q(z)} .
$$

To establish that this function has the requisite properties note that its only singularities occur from the zeros of the denominator. These are just the points $\mu_{i}$ of case III. Thus a sufficient condition for the solution is that

$$
M\left(\mu_{i}\right)=0 .
$$

But this is from $(8.7)$ and $(8.3,8.4)$ explicitly

$$
\begin{aligned}
M\left(\mu_{i}\right) & =\int \frac{f_{1}\left(v^{\prime}\right) d v^{\prime}}{v^{\prime}-\mu_{i}}=\int \frac{f d v^{\prime}}{v^{\prime}-\mu_{i}}-\sum_{j} a_{j} \int \frac{y_{j}(v) d v}{v-\mu_{i}} \\
& =\int \frac{f d v^{\prime}}{v^{\prime}-\mu_{i}}+\sum a_{j} \int z_{i}(v) y_{j}(v) d v=0
\end{aligned}
$$

so that these conditions are satisfied.

The solution of the initial value problem for (8.1) when initially $y(v, 0)=y_{0}(v)$ is now given by

$$
y(v, t)=\sum a_{i} e^{-i k \mu_{i} t} y_{i}(v)+\int A(\mu) e^{-i k \mu t} y_{\mu}(v) d \mu
$$

where

$$
a_{i}=\frac{1}{c_{i}} \int z_{i}(v) y_{0}(v) d v=-\frac{\int y_{0}(v) d v /\left(v-\mu_{i}\right)}{\int n(v) d v /\left(v-\mu_{i}\right)^{2}}
$$

and

$$
\begin{aligned}
A(\mu) & =\frac{1}{c_{\mu}} \int z_{\mu}(v) y_{0}(v) d v \\
& =\frac{1}{\lambda^{2}(\mu)+\pi^{2} n^{2}(\mu)}\left[\lambda(\mu) y_{0}(\mu)-n(\mu) P \int \frac{n(v) y_{0}(v) d v}{v-\mu}\right] .
\end{aligned}
$$

The above expansion reduces for $t=0$ to the expansion in terms of the discrete and continuous eigenfunction distributions determined above. The intimate connection of this procedure with that of the 
Laplace transform method can be seen by using the two-sided Laplace transform to solve (8.1) directly. For this purpose let

$$
\begin{aligned}
y_{+}(v, t) & =y(v, t), t>0, & y_{-}(v, t) & =0, t>0, \\
\lim _{t \rightarrow 0^{+}} y(v, t) & =y(v, 0), & \lim _{t \rightarrow 0^{-}} y_{-}(v, t) & =y(v, 0), \\
y_{+}(v, t) & =0, t<0, & y_{-}(v, t) & =y(v, t), t<0 .
\end{aligned}
$$

Now $y_{+}(v, t)$ satisfies (8.11) except near $t=0$ and since

$$
\int_{0-\epsilon}^{0+\epsilon} \frac{\partial y_{ \pm}}{\partial t} d t=y_{ \pm}(v, \epsilon)-y_{ \pm}(v,-\epsilon)= \pm y(v, 0)
$$

we can replace (8.1) by

$$
\frac{\partial y_{ \pm}}{\partial t}+i k v y_{ \pm}= \pm y(v, 0) \delta(t)-i k n(v) \int_{-\infty}^{\infty} y_{ \pm}\left(v^{\prime}, t\right) d v^{\prime} .
$$

If the Laplace transforms $h_{ \pm}$of $y_{ \pm}$are defined by

$$
h_{ \pm}(v, z)=\int_{-\infty}^{\infty} y_{ \pm}(v, t) e^{i z t} d t
$$

so that (8.12) becomes

$$
(k v-z) h_{ \pm}=\mp i y_{0}(v, 0)-k n(v) \int_{-\infty}^{\infty} h_{ \pm}\left(v^{\prime}, z\right) d v^{\prime}
$$

from which it follows that

$$
h_{ \pm}(v, z)=\mp i y(v, 0)-\frac{k n(v)}{k v-z} \int_{-\infty}^{\infty} h_{ \pm}\left(v^{\prime}, z\right) d v^{\prime}
$$

and that

$$
h_{ \pm}\left(v^{\prime}, z\right) d v^{\prime}= \pm \frac{\int y(v, 0) d v /(k v-z)}{\left[1+k \int n(v) d v /(k v-z)\right]} .
$$

Using the complex inversion formulas

$$
y_{ \pm}(v, t)=\frac{1}{2 \pi} \int_{C_{ \pm}} h_{ \pm}(v, z) e^{-i z t} d z
$$


where $C_{ \pm}$run parallel to the real axis above and below all the singularities of $h_{ \pm}(v, z)$ respectively, one finds

$$
y(v, t)=y_{+}(v, t)-y_{-}(v, t) .
$$

If these contour integrals are now evaluated taking into account the zeros of the denominators of the integrands and the form of $z$ on the paths, it is readily verified that the result is identical to that given by (8.11) from the normal mode approach.

While a detailed discussion of the application of these ideas to quantum scattering and field theory is not possible here, their relevance can be seen from consideration of Schrödinger's equation for a simple nonlocal interaction. Specifically, consider

$$
i \frac{\partial \psi}{\partial t}=-\frac{\nabla^{2} \psi}{2 m}+V(r) \int V\left(r^{\prime}\right) \psi\left(r^{\prime}\right) d^{3} r^{\prime}
$$

and restrict attention to $t>0$. Let $\Psi$ be the Laplace transform of $\psi$ as defined by (8.13), let $\psi_{0}$ denote the value of $\psi$ at $t=0$, and define $\bar{\Psi}$ as the Fourier transform of $\Psi$ with respect to $r$, letting $\Psi_{0}$ be the transformed initial value of $\psi_{0}$, by means of

$$
\bar{\Psi}(p, z)=\frac{1}{(2 \pi)^{3 / 2}} \int \Psi(r, z) e^{-i p \cdot r} d^{3} r .
$$

If one sets $E(p)=p^{2} / 2 m$, the application of the double transform yields the integral equation

$$
[E(p)-z] \bar{\Psi}-\bar{V}(\boldsymbol{p}) \int \bar{V}\left(\boldsymbol{p}^{\prime}\right) \bar{\Psi}\left(\boldsymbol{p}^{\prime}, z\right) d^{3} \boldsymbol{p}^{\prime}=-i \Psi_{0} .
$$

This is readily solved to yield

with

$$
\bar{\Psi}(p, z)=-i \frac{\left[\Psi_{0}(p)+C(z) \bar{V}(p)\right]}{E(p)-z}
$$

$$
C(z)=\int \frac{\bar{V}\left(p^{\prime}\right) \Psi_{0}\left(p^{\prime}\right) d^{3} p^{1}}{E\left(p^{\prime}\right)-z} /\left[1-\int \frac{\bar{V}^{2}\left(p^{\prime}\right) d^{3} p^{1}}{E\left(p^{\prime}\right)-z}\right] .
$$

Application of the inverse Laplace transform then gives

$$
\bar{\psi}(p, t)=\frac{1}{2 \pi i} \int_{C_{+}}\left\{\frac{\Psi_{0}+C(z) \bar{V}(p)}{E(p)-z}\right\} e^{-i z t} d z .
$$

The similarity of (8.14) with (8.16) and (8.15) with (8.17) is apparent. To continue the discussion, one displaces the contour $C+$ down 
as far as possible taking into account the pole at $z=E(p)$, the singularities of the denominator of the coefficient of $\bar{V}(p)$ in (8.17) (these are readily seen to be the bound states), the existence of a branch cut along the positive real axis which occurs, and one considers, in addition, the final value problem. The interested reader is referred to the lectures [16] of Case where the limiting processes necessary to approximate a wide class of potentials by kernels of the type considered are carried through.

9. Wiener-Hopf and Cauchy singular integral equations. Next, we will note how these same ideas can be used to include the theory of the Wiener-Hopf integral equation under the theory of singular integral equations. The observations below are unpublished and due to conversations between $\mathrm{K}$. M. Case and the author. For simplicity we will limit consideration to the equation

$$
\phi(x)=\int_{0}^{\infty} K(x-y) \phi(y) d y
$$

but the extensions to the general situation are immediate.

METHOD I. Introduce the Heaviside unit function and write (9.1) as

$$
\phi(x)=\int_{-\infty}^{\infty} K(x-y) \phi(y) h(y) d y .
$$

Assuming that Fourier transforms exist and are denoted by the corresponding barred functions, this can be written as

$$
\Phi(k)=\bar{K}(k) \int_{-\infty}^{\infty} \bar{\phi}\left(k^{\prime}\right) \bar{h}\left(k-k^{\prime}\right) d k^{\prime} .
$$

However, from the theory of generalized functions, (see for example page 43 of Lighthill [VII]), the Fourier transform $h(k)$ of $h(t)$ exists as a distribution and is given by

$$
\frac{1}{2} \delta(k)+\frac{1}{2 \pi i k} .
$$

Insertion then yields the singular integral equation

$$
\Phi(k)=\bar{K}(k)\left\{\frac{1}{2 \pi i} \int_{-\infty}^{\infty} \frac{\Phi\left(k^{\prime}\right) d k^{\prime}}{k-k^{\prime}}+\frac{\Phi(k)}{2}\right\} .
$$

METHOD II. Introduce the function 


$$
\begin{aligned}
& \psi(x)=\phi(x), \quad 0 \leqq x<\infty, \\
& =0, \quad-\infty<x<0 \text {, }
\end{aligned}
$$

so that (9.1) becomes

$$
\phi(x)=\int_{-\infty}^{\infty} K(x-y) \psi(y) d y .
$$

Defining the functions

$$
\bar{\phi}_{ \pm}(k)= \pm \frac{1}{2 \pi} \int_{0}^{ \pm \infty} \phi(x) e^{-i k x} d x
$$

equation (9.3) transforms into

$$
\bar{\phi}(k)=\bar{\phi}_{+}(k)+\bar{\phi}_{-}(k)=(2 \pi)^{1 / 2} \bar{K}(k) \bar{\phi}_{ \pm}(k) .
$$

Let $N^{+}(z)$ be the analytic continuation of $\Phi_{+}$to the upper half plane and $N^{-}(z)$ be that of $-\Phi_{-}$to the lower half plane so that

$$
N^{+}(k)-N^{-}(k)=\bar{\phi}_{+}(k)+\phi_{-}(k)=\bar{\phi}(k) .
$$

Now consider the analytic function

$$
N(z)=\frac{1}{2 \pi i} \int_{-\infty}^{\infty} \frac{\phi\left(k^{\prime}\right) d k^{\prime}}{k^{\prime}-z} .
$$

According to the Plemelj formulas we have

$$
N^{ \pm}(k)=\bar{\phi}_{ \pm}(k)=\frac{1}{2 \pi i} P \int_{-\infty}^{\infty} \frac{\Phi\left(k^{\prime}\right) d k^{\prime}}{k^{\prime}-k} \pm \frac{1}{2} \Phi(k) .
$$

Insertion of (9.5) into (9.4) yields (9.2)

METHOD III. Introducing a convergent factor (9.1) becomes

$$
\Phi(k)=\lim _{\iota \rightarrow 0} \int_{0}^{\infty} K(x-y) e^{-\iota y} \phi(y) d y
$$

with a Fourier transform given by

$$
\Phi(k)=\frac{\bar{K}(k)}{2 \pi i} \int_{-\infty}^{\infty} \frac{\phi\left(k^{\prime}\right) d k^{\prime}}{k^{\prime}-(k+i \epsilon)} .
$$

Allowing $\epsilon \rightarrow 0$ one obtains

$$
\Phi(k)=\bar{K}(k)\left\{\frac{1}{2 \pi i} P \int_{-\infty}^{\infty} \frac{\Phi(k) d k}{k^{\prime}-k}+\frac{\Phi(k)}{2}\right\}
$$

which again is the same as (9.2). 
While the above has been done formally, it is not a difficult task to state sufficient conditions for the validity of the three methods. We will not enter into this explicitly but shall refer the reader to the papers by Sparenberg [93; 94], Widom [103] and Krein [52] Krein and Gohberg [54] for the rigorous theory of these equations and to the report by Bremmerman and Durand [5] who discuss a generalization of the problem of analytic continuation for distributions (cf. also Köthe [50] and Tillmann [95]).

The examples we have briefly sketched serve to illustrate how a unified approach to many apparently unrelated problems of mathematical physics can be achieved by the use of distributions and the methods due to Plemelj in which the concept of self- or non-selfadjointness does not enter explicitly.

10. Variational principles for non-self-adjoint problems. It is well known that variational principles such as those associated with the names of Rayleigh-Ritz, Dirichlet, Glerkin, Treftz, and Schwinger have proved valuable both theoretically and practically for many self-adjoint problems of both applied and appliable mathematics involving compact domains. On occasion they have even been used with moderate success for non-self-adjoint compact problems but often here if the natural bilinear form of the problem is used, the stationary points obtained are not minima and a convergence theory of successive improvement is often lacking. For noncompact selfor non-self-adjoint problems the situation is much more complicated and a general theory is lacking. It is, of course, true that variational principles such as those of Schwinger, Hulthén and Kohn, and MacFarlane have been used for such problems but the absence of a firm theoretical basis does not make it surprising that they have often yielded unsatisfactory results. In fact, at this time, only the principle due to Garabedian [30] which we will describe shortly, seems to rest on firm mathematical grounds.

While we do not intend to enter into a detailed discussion of each of the principles that have been used for noncompact problems, we will attempt to summarize their current status.

Perhaps the most widely used of these is that attributed to Schwinger which in its usual form yields a value of a transmitted or reflected scattering amplitude at its stationary points and which depends upon the real symmetry of the free space Green's function of the Helmholtz equation governing the scattering problem in scalar theory or the interactionless state in quantum theory.

From an abstract point of view, one obtains the Schwinger variational principle for the equations 


$$
\begin{aligned}
A x & =f, \\
A^{*} y & =g,
\end{aligned}
$$

whose solutions are, say, $x_{0}, y_{0}$ when, with respect to some inner product, $(A x, y)=\left(x, A^{*} y\right)$, by considering the expression

$$
J=\left(A\left(x-x_{0}\right), y-y_{0}\right)=(A x, y)-(f, y)-(x, g)-\left(A x_{0}, y_{0}\right),
$$

which is clearly stationary with respect to independent variations of $x, y$. Now if in the equivalent expression

$$
H=(A x, y)-(f, y)-(x, g),
$$

one requires that $H$ at its stationary points be independent of the scale of $x$ and $y$; i.e. invariant under $x \rightarrow \tau x^{\prime}$ and $y \rightarrow \mu y^{\prime}$, one readily finds that alternately

$$
H=-\frac{(f, y)(x, g)}{(A x, y)}
$$

will be stationary if the equations $A\left(\tau x_{0}^{\prime}\right)=f, A^{*}\left(\mu y_{0}^{\prime}\right)=g$ hold, provided that $\left(x_{0}, g\right) \neq 0,\left(f, y_{0}\right) \neq 0$. In this form the value of $H$ at a stationary point admits a physical interpretation in scattering problems. The form of $H$ above suggests that consideration of its reciprocal plus an appropriate normalization and rotation of coordinates should yield a stationary expression similar to $\left(x^{\prime}\right)^{2}-\left(y^{\prime}\right)^{2}$ so that the saddle nature would be apparent. Actually while this is in principle true the situation is more complicated in that $H$ is usually complex valued. None the less, the process can be carried through by working with the cartesian product of two Hilbert spaces and as Dolph and Ritt [21] have shown, the property that makes this demonstration possible is the dissipative nature of the operators $A$ for scattering processes.

In particular for the case of one-dimensional quantum scattering one can consider for a positive (or negative definite potential) $V(x)$ the symmetrized operator on $L_{2}(-\infty, \infty)$ defined by

$$
K \phi=\frac{1}{2 k i} \int_{\infty}^{\infty} U(x) e^{i k|x-y|} U(y) \phi(y) d y
$$

where $U^{2}(x)=V(x) \geqq 0$.

THEOREM 10.2. (a) $K$ is a bounded compact linear operator whose norm satisfies

$$
\|K\|<\frac{1}{2 k}\|V(x)\|^{2}
$$


(b) If $\phi_{0}$ is defined by $\phi_{0}(x)=U(x) e^{i k x}$, then

$$
(K \phi, \psi)-(\phi, K \psi)=\frac{1}{2 k i}\left\{\left(\phi, \phi_{0}\right)\left(\phi_{0}, \psi\right)+\left(\phi, \phi_{0}\right)\left(\bar{\phi}_{0}, \psi\right)\right\}
$$

for all $\phi, \psi$ in $L_{2}(-\infty, \infty)$.

(c) The operators $A=I-K, A^{*}=I-K^{*}$ have bounded inverses in the Hilbert space $H$ consisting of the orthogonal complement of the null space of $I-K$.

(d) The Fredholm equations

$$
A \phi=\phi_{0}, \quad A^{*} \bar{\psi}=\phi_{0},
$$

have a unique solution $\phi_{1}$ and $\psi_{1}$ in $H$ such that

$$
\left(\phi_{1}, \phi_{0}\right)=\left(\phi_{0}, \Psi_{1}\right) \text {. }
$$

(e) If $\|V(x)\|^{2}<2 k$ then $\left(\phi_{1}, \phi_{0}\right) \neq 0$.

In the above, property (b) for $\psi=\phi_{0}$ is identical to the relation (7.1) and is the dissipative property while (c) and (e) are special instances of Theorem (7.3).

In these terms the Schwinger variational principle for the transmission coefficient consists of the problem of rendering

$$
J=(A \phi, \bar{\psi})
$$

stationary subject to the normalizing constraint

$$
\left(\phi_{1} \phi_{0}\right)\left(\phi_{0}, \Psi\right)=1
$$

or, in terms of the Lagrange multiplier rule, the expression

$$
J_{\lambda}=J-\lambda\left(\phi, \phi_{0}\right)\left(\phi_{0}, \bar{\psi}\right) \text {. }
$$

For this we have the precise statement:

THEOREM 10.2. There exists one value of $\lambda, \lambda_{1}$ such $J_{\lambda}$ can be made stationary. $\lambda_{1}$ is equal to the value of $J$ when $J$ is made stationary subject to the constraint (10.2). Simultaneously $\lambda_{1}=1 /\left(\phi_{1}, \phi_{0}\right)$. The pair $\phi_{1}$, $\psi$ make $J$ stationary subject to (10.2) if and only if there exists an $\alpha \neq 0$ such that $\phi=\alpha \lambda_{1} \phi_{1},=(1 / \alpha) \lambda_{1} \psi_{1}$.

The above principle can be reformulated as a quadratic problem by introducing the space $H=H \oplus H$ whose elements $\phi$ are of the form $[\phi, \psi]$, where $\phi, \psi$ are in $H$, and the inner product is given by

$$
\left\langle\phi^{\prime}, \phi^{\prime \prime}\right\rangle=\left(\phi^{\prime}, \phi^{\prime \prime}\right)+\left(\psi^{\prime}, \psi^{\prime \prime}\right) \text {. }
$$

Now if $\boldsymbol{C}$ is the mapping of $H$ into $H$ defined by $C \boldsymbol{\phi}=\boldsymbol{C}[\phi, \psi]=[\psi, \phi]$ then $C^{2}=I,\langle C \phi, C \psi\rangle=\langle\psi, \phi\rangle, C(\lambda \phi)=\bar{\lambda} C \phi$. If 


$$
\phi_{0}=\left[\phi_{0}, \phi_{0}\right], \quad \psi_{0}=\left[\phi_{0},-\bar{\phi}_{0}\right]
$$

then

$$
\begin{aligned}
& C \phi_{0}=\phi_{0}, \\
& C \psi_{0}=-\psi_{0} .
\end{aligned}
$$

If $L$ is the mapping of $H$ into $H$ defined by

$$
L \boldsymbol{\phi}=L[\phi, \psi]=[A \phi, A \psi]
$$

then $L$ is a linear operator; $L^{*}$ the conjugate of $L$ is given by $L^{*} \phi$ $=\left[A^{*} \phi, A^{*} \psi\right]$ and $L^{*} \mathrm{C}=C L$. Finally, if we now define $J=2\langle L \boldsymbol{\phi}, \boldsymbol{C} \phi\rangle$ and consider the problem of making this stationary subject to the constraint

$$
\left\langle\phi, \phi_{0}\right\rangle^{2}-\left\langle\phi, \psi_{0}\right\rangle^{2}=\psi
$$

or, equivalently, consider the free problem of making

$$
J_{\lambda}=J-\lambda\left\{\left\langle\phi, \phi_{0}\right\rangle^{2}-\left\langle\phi, \psi_{0}\right\rangle^{2}\right.
$$

stationary, we have the results:

THEOREM 10.3. There is a unique value of $\lambda, \lambda_{1}$ such that $J_{\lambda}$ can be made stationary. $\lambda_{1}$ is the stationary value of $J$ subject to the constraint (10.2). $\lambda_{1}$ is the same as in Theorem (10.1). $\phi_{1}=\left[\lambda_{1} \phi_{1}, \lambda_{1} \psi_{1}\right]$ is a stationary point, and all other stationary points are of the form $\phi(\alpha)$ $\left[\alpha \lambda_{1} \phi_{1},(1 / \alpha) \lambda_{1} \psi_{1}\right], \alpha \neq 0$.

From the equivalence of Theorems (10.2) and (10.1) we have the following characterization:

TheOREM 10.4. Let $\phi_{s}=\alpha \lambda_{1} \phi_{1}, \psi_{s}=(1 / \alpha) \lambda_{1} \psi_{1}$ be a pair of functions which make $J=(A \phi, \Psi)$ stationary subject to the constraint $\left(\phi, \phi_{0}\right)\left(\phi_{0}, \bar{\phi}\right)$ $=1$. Then if $\phi$ and $\psi$ are varied about $\phi_{s}, \psi_{s}$ subject to the constraint then

(a) If $|\alpha| \neq 1$, and if $\phi+\Psi$ is held constant, then $\operatorname{Im} J$ will have $a$ local maximum at $\phi_{s}, \psi_{\mathrm{s}}$. If $\phi-\bar{\psi}$ is held constant, Im $J$ will have a local minimum at $\phi_{s}, \psi_{s}$.

(b) If $|\alpha|=1$, and if $\phi+\Psi$ is held constant, then $\operatorname{Im} J$ will have a global maximum.

Similar characterizations can be obtained for the reflection amplitude in this case and the theory can be generalized to three dimensions. While Dolph [19] has shown that similar behavior can be expected from other than quantum mechanical scattering problems, the occurrence of Fredholm integral equations of the first kind makes it difficult to establish similar results rigorously there. In addition 
the above suffers from the requirement of definiteness of the given potential and it may be conjectured that this limitation can be removed only by consideration of the theory of Hilbert spaces with an indefinite metric whose theory we shall comment on shortly.

The real symmetry of a class of dissipative differential operators can be used to provide a formal extension of the Rayleigh-Ritz principle. Thus for the differential equation with $q(x)$ real but subject to a radiation condition at infinity such as for the problem treated by Phillips [83]; namely,

$$
-y^{\prime \prime}+[q(x)-\lambda] y=0,
$$

$$
y(a)=0, \quad \lim _{x \rightarrow \infty} \alpha y(x)+\beta y^{\prime}(x)=0, \quad \alpha, \beta \text { complex },
$$

the expression

$$
R(y)=\int_{a}^{\infty} y\left[-y^{\prime \prime}+q(x) y\right] d x / \int_{a}^{\infty} y^{2} d x
$$

is stationary as first observed and used by MacFarlane [71]. For this case the eigenfunctions are orthogonal with respect to a real inner product (cf. also Sommerfeld [XIII, Appendix II, Chapter V] in spite of their complex values and it is a consequence of the theory of Phillips that all the complex eigenvalues will be confined to a halfplane. (Alternately, in terms of a hermitian inner product, the adjoint eigenfunction corresponding to the complex conjugate of a given eigenfunction is easily seen to have the property that its complex conjugate is identical to the eigenfunction corresponding to (10.3).) The confinement to a half-plane suggests the possibility of obtaining a characterization of the imaginary parts of the eigenvalues in this case for if an expansion theory existed in terms of the real orthonormal eigenfunctions, say $y=\sum_{1}^{\infty} z_{i} y_{i}(x), R(y)$ would reduce to

$$
R(y)=R\left(z_{1}, z_{2}, \cdots\right)=\sum_{1}^{\infty} \lambda_{i} z_{i}^{2} / \sum z_{i}^{2} .
$$

While the absence of precise expansion theorems for even the second order case are missing on the infinite interval, it is reasonable to conjecture that a sequential min-max characterization of the imaginary part of the eigenvalues of a dissipative differential operator does in fact exist, under suitable if as yet unspecified conditions. In particular for the finite dimensional analogue, that of finding the stationary points of a quadratic form

$$
Q(z)=\sum a_{i j} z_{i} z_{j}
$$


with complex coefficients possessing real symmetry $a_{i j}=a_{j i}$ subject to the "real" normalization condition

$$
H(z)=\sum z_{i}^{2}=1
$$

such a principle does in fact exist if the form is assumed diagonalizable and if its spectrum is confined to a half-plane as it would be if it is to be considered a reasonable approximation to a dissipative operator. In particular, we have the following theorem (Dolph, Marx, and McLaughlin [20]).

TheOREM. Let $Q(z)=R(x, y)+i I(x, y)$ be a complex symmetric quadratic form having a spectral theorem, and let the imaginary parts of the characteristic values of $Q$ be positive. The lowest imaginary part is the smallest value attained by the maximum of $I(x, y)$ for vectors $\mathbf{x}, \mathbf{y}$ satisfying the conditions $0 \leqq I(x, y) \leqq T$ (where $T$ is the imaginary part of the trace of $Q), H(z)=1$ and $(n-1)$ arbitrary, linear, homogeneous constraints with real coefficients on the components of the $2 n$-dimensional vectors $\mathbf{x}, \mathbf{y}$. If $\mathbf{u}^{k}, \mathbf{v}^{k}, k=1, \cdots, m-1$ are $(m-1)$ vectors for which

$$
I\left[u^{k}, v^{k}\right]=\mu_{k}, \quad k=1, \cdots,(m-1)
$$

and also

$$
\left(u^{i}+i v^{i}, u^{k}+i v^{k}\right)=\delta_{i j},
$$

then $\mu_{m}$ is the smallest value attained by the maximum of $I(x, y)$ for vectors satisfying the conditions $0 \leqq I(x, y) \leqq T, H(z)=1$,

$$
\left(\mathrm{x}+i \mathrm{y}, \mathrm{u}^{k}+i \mathrm{v}^{k}\right)=0, \quad k=1, \cdots,(m-1)
$$

and $(n-m)$ arbitrary linear homogeneous constraints.

The reformulation of this problem independent of the dimension of the underlying space and its generalization to Hilbert space are open.

An interesting but as yet largely unexploited variational principle has been developed by Garabedian [30]. (Cf., however, the report by Wilcox and Hartman [114].) One defines the norm

$$
\|u\|^{2}=\lim _{r \rightarrow \infty} \int_{|x|-r}\left\{|u|^{2}+\left|\frac{\partial u}{\partial \eta}\right|^{2}\right\} d^{2} \tau
$$

for solutions of the Helmholtz equation in a region exterior to a closed domain $D$. Now if $v$ is the solution of

$$
\nabla^{2} v+v=0
$$

satisfying 


$$
\lim _{r \rightarrow \infty} \int_{|x|=r}\left|\frac{\partial v}{\partial r}-i v\right|^{2} d^{2} \tau=0
$$

and taking the value $f$ on the $B$, the boundary of $D$, and if $w$ is the solution of (10.4) and (10.5) taking the value $f^{*}$ on $B$, then the function

$$
u_{0}=\frac{v+w^{*}}{2}
$$

is characterized by the property that among all the solutions of (10.4) with the boundary values $f$, it has the smallest norm,

$$
\left\|u_{0}\right\|=\min \|u\| \text {. }
$$

While this principle clearly has the advantage of being a minimum rather than a stationary principle, the necessity of having to consider the solutions $v$ and $w$ makes its application difficult.

The situation is more confused in regard to more straight-forward analogues of the Dirichlet principle for compact problems and any attempt to pass from the Laplace equation to the Helmholtz equation directly leads, in the words of $\mathrm{H}$. Weyl [102], to integrals of doubtful convergence. The only principle which has been used extensively in calculations is that due to Hulthén-Kohn $[40 ; 48 ; 49]$, which for the case of Schrödinger's time independent equation

$$
\left[\nabla^{2}+k^{2}-V(r)\right] \psi=0
$$

takes the form

$$
\text { (10.6) } 4 \pi A_{t}^{2} f\left(k_{1},-k_{2}\right)=4 \pi A_{t}^{2} f_{l}\left(k_{1},-k_{2}\right)+\int \psi_{2}\left\{\nabla^{2}+k^{2}-V\right\} \psi_{1} d^{2} r
$$

Here $\psi_{i}$ represents a wave function incident along the direction $k_{i}$ and is assumed to be of the asymptotic form

$$
\psi_{i} \sim A_{i}\left\{e^{i k_{i} \cdot r}+f_{t}\left(k_{i}, k\right) \frac{e^{i k r} r}{r}\right\}
$$

where $f_{t}\left(k_{i}, k\right)$ represents the trial scattering amplitude in the direction $k$ of a plane wave incident along $k_{i}$. In (10.6) the term on the left-hand side denotes the true but unknown scattering amplitude of scattering from the direction $k_{1}$ to $-k_{2}$. The second term on the righthand side is stationary for arbitrary variations of $\psi_{i}$ with the above asymptotic form and is actually zero at its stationary points so that 
(10.6) may be thought of as a variational principle for trial functions with correct asymptotic behavior but probably false scattering amplitudes. Now if the Schrödinger operator is eliminated by the use of the free space Green function of the Helmholtz equation, is readily seen to be equivalent to the principle of Schwinger. That is, the correct wave function $\psi_{i}$ satisfies the integral equation

$$
\psi_{i}(\boldsymbol{r})=e^{i k_{i} / \boldsymbol{r}}-\int \frac{e^{i k\left|\boldsymbol{r}-\boldsymbol{r}^{\prime}\right|}}{4 \pi\left|\boldsymbol{r}-\boldsymbol{r}^{\prime}\right|} V\left(\boldsymbol{r}^{\prime}\right) \psi_{i}\left(\boldsymbol{r}^{\prime}\right) d^{3} \boldsymbol{r}^{\prime}
$$

which, with the well-known relation (cf. Morse and Feshbach [XI, Part II])

$$
f\left(k_{1},-k_{2}\right)=-\frac{1}{4 \pi} \int e^{i k_{\mathbf{2}} \cdot \boldsymbol{r}^{\prime} V\left(\boldsymbol{r}^{\prime}\right) \psi_{1}\left(\boldsymbol{r}^{\prime}\right) d r^{\prime}}
$$

can be written as

$$
\psi_{i}(\boldsymbol{r})=-\int G\left(\boldsymbol{r}, \boldsymbol{r}^{\prime}\right) V\left(\boldsymbol{r}^{\prime}\right) \psi_{i}\left(\boldsymbol{r}^{\prime}\right) d \boldsymbol{r}^{\prime}
$$

where

$$
G=\frac{1}{4 \pi f\left(k_{1},-k_{2}\right)} e^{i k_{1} \cdot r} e^{i k_{2} \cdot r^{\prime}}+\frac{e^{i k\left|r-r^{\prime}\right|}}{4 \pi\left|r-r^{\prime}\right|} .
$$

Now it should be noted that whatever $\psi_{i}$ is used in the right-hand side of (10.7), the left-hand side is a wave function whose scattering amplitude from $k_{1}$ to $-k_{2}$ is correctly given by $f\left(k_{1},-k_{2}\right)$. Thus if one takes trial functions $\psi_{i}, i=1,2$,

$$
\psi_{i}=-\int G\left(\boldsymbol{r}, \boldsymbol{r}^{\prime}\right) V\left(\boldsymbol{r}^{\prime}\right) \phi_{i}\left(\boldsymbol{r}^{\prime}\right) d \boldsymbol{r}^{\prime}
$$

it will follow that $f_{t}\left(k_{1},-k_{2}\right)=f\left(k_{1},-k_{2}\right)$ so that $I\left(k_{1},-k_{2}\right)=0$. Substitution shows that this, however, is

$$
\begin{aligned}
\int \psi_{2} U \phi_{1} d r & +\iint \psi_{2}(\boldsymbol{r}) V(\boldsymbol{r}) \frac{e^{i k\left|\boldsymbol{r}-\boldsymbol{r}^{\prime}\right|}}{4 \pi\left|\boldsymbol{r}-\boldsymbol{r}^{\prime}\right|} V\left(\boldsymbol{r}^{\prime}\right) \phi_{1}\left(\boldsymbol{r}^{\prime}\right) d^{3} r d^{3} r^{\prime} \\
& +\frac{1}{4 \pi f\left(k_{1},-k_{2}\right)}\left(\int \psi_{2} V(\boldsymbol{r}) e^{i k_{1} \cdot \boldsymbol{r}} d^{3} r\right)\left(\int \phi_{1}(\boldsymbol{r}) e^{i k_{2}^{\prime} \boldsymbol{r}} V(\boldsymbol{r}) d^{3} r\right)
\end{aligned}
$$

and when this is solved for $1 / 4 \pi f\left(k_{1},-k_{2}\right)$ it yields the Schwinger variational principle 


$$
\begin{aligned}
& \frac{1}{4 \pi f\left(k_{1},-k_{2}\right)} \\
& =-\frac{\int \psi_{2} V \phi_{1} d^{3} r+\iint \psi_{2} V(\boldsymbol{r}) \frac{e^{i k\left|r-\boldsymbol{r}^{\prime}\right|}}{4 \pi\left|r-\boldsymbol{r}^{\prime}\right|} V\left(\boldsymbol{r}^{\prime}\right) \phi_{1}\left(\boldsymbol{r}^{\prime}\right) d^{3} r d^{3} \boldsymbol{r}^{\prime}}{\left(\int \psi_{2} V(r) e^{i k_{1} \cdot \boldsymbol{x}} d^{3} r\right)\left(\int \phi_{1}(\boldsymbol{r}) e^{i k 2 \cdot r} V(\boldsymbol{r}) d^{3} \boldsymbol{r}\right)}
\end{aligned}
$$

and is therefore subject to the same type of mathematical criticism. Often in practice it seems to be preferable since it does not involve such complicated integrals. These same criticisms also apply to the near-field generalizations of these same principles as developed by Levine [62] and Dolph and Altshuler [23].

In an attempt to overcome the difficulties which occur because the indefinite natural Hilbert metric associated with many elliptical differential equations such as Schrödinger's and Helmholtz's, Nevanlinna in a series of papers [79] developed a theory of Hilbert spaces with an indefinite metric. Recently Krein and Iohvidov [52] have also systematically studied the spectrum of linear operators in these spaces. One of Nevanlinna's results was a generalized Dirichlet principle appropriate to self-adjoint elliptic operators on a bounded domain and this principle has been used by Louhivarra [68] to give a discussion of the existence and uniqueness of the solution to the generalized Dirichlet boundary value problems for such second order equations. Subsequent to this, Browder $[6 ; 7]$ and Littman [66] generalized his results to higher order equations and at the same time removed the restriction of self-adjointness and the limitation to bounded domains. Recently, Louhivarra [69] examined the relationship between the results of Browder and Littman and gave a characterization of the range of bounded linear transformations in indefinite Hilbert spaces. At the same time, Hilbert spaces with an indefinite metric are playing an increasing role in physical theories following the success achieved by Bleuer [2] and Gupta [36] in the theory of quantum electrodynamics. An introduction to the way such spaces are introduced in the physical theories as well as a discussion of some of the difficulties caused by the existing theory can be found in the article of Prandit [86].

In this approach, in order to solve the Dirichlet problem for a differential operator $L u=f$ in an open domain $D$ of three dimensional space with $u=g$ on $B$, the boundary of this domain, one first extends the function $g$ to the interior of $D$ and replaces the above problem by that 
of finding a $v$ such that $L v=f_{1}$ in $D$ and $v=0$ on $B$ where the relationships are $v=u-g$ and $f_{1}=f L g$. In order to obtain a weak solution one notes that there is a bilinear form associated with the second problem obtained by integration by parts, that is, under suitable restrictions on the coefficients.

$$
\begin{aligned}
(L v, w) & =\left(f_{1}, w\right) \\
& =\left(v, L^{*} w\right)=B(v, w) .
\end{aligned}
$$

(The bilinear form is not uniquely determined except in the second order case.) Next the known linear functional

$$
F(w)=\left(f_{1}, w\right)-B(g, w)
$$

is considered and if this can be shown to be bounded, then there exists a unique $z$ such that $F(w)=B(z, w)=\left(f_{1}, w\right)-B(g, w)$. The solution to the generalized problem is then given by $v=z+g$. More precisely, in the second-order case, let $C^{(2)}$ be the linear space of twice continuously differentiable complex-valued functions defined over the closure of $D$ and let $H_{2}$ be the completion of $C^{(2)}$ with respect to the norm

$$
\|u\|_{2}^{2}=\int|u|^{2} d^{3} x+\int|\nabla u|^{2} d^{3} x
$$

while $H_{2}^{0}$ is the completion with respect to the same norm of the subset of functions from $C^{2}$ which have compact support in $D$. The generalized Dirichlet problem then consists of being given a $g$ in $\mathrm{H}_{2}$ and an $f_{1}$ in $L_{2}$ to find a $v$ in $H_{2}$ such that $v-g$ belongs to $H_{2}^{0}$ and for all $w$ in $H_{2}^{0}$,

$$
B(v-g, w)=\left(f_{1}, w\right)-B(g, w) .
$$

Since the bounded bilinear form can be extended boundedly to functions in $H_{2}$, the form $B(z, w)$ can be written as

$$
B(z, w)=(A z, w)
$$

where $A$ is a bounded operator on $H_{2}^{0}$ and the usual $L_{2}$ inner product occurs in the right-hand side. If $g$ is taken to be zero the generalized problem can then be stated as that of finding a $z$ in $H_{2}^{0}$ such that

$$
\left(f_{1}, w\right)=(A z, w)=\left(z, A^{*} w\right)
$$

for all $w$ in $H_{2}^{0}$ where $A^{*}$ denotes the adjoint of $A$. A necessary condition for the solvability is clearly that $f$ be orthogonal in the $L_{2}$ sense to all elements of the null space of $A^{*}$. This condition is, however, not sufficient and in order to obtain a sufficient condition, it is neces- 
sary to obtain a somewhat larger space than the above null space. This is accomplished in case the bilinear form $B(z, w)$ is hermitian, the case considered by Nevanlinna [79] and applied by Louhivarra [68] by decomposing $H_{2}^{0}$ with the aid of $A$. Since $A$ is self-adjoint, spectral theory implies that there will be a resolution of the identity such that

$$
A z=\int_{-1}^{1} \lambda d E_{\lambda} z
$$

There is no loss in generality in assuming the range to be $(-1,1)$. In order to obtain a self-adjoint operator in the general case, Littman [66] considers the hermitian ${ }^{16}$ part of $B$, namely,

$$
\tilde{B}(z, w)=B(z, w)+B^{*}(w, z)
$$

for which there will be associated a self-adjoint operator $\tilde{A}$ defined on $H_{2}^{0}$ such that

$$
\widetilde{B}(z, w)=(A z, w)=(z, A w)
$$

for all $z, w$ in $H_{2}^{0}$. This operator will of course admit a spectral representation in the form (10.8) so that in all cases it is possible to define subspaces $S_{+}$and $S_{-}$as the respective ranges of the operators

$$
\int_{\epsilon}^{1} d E_{\lambda} \text { and } \int_{-1}^{-\bullet} d E_{\lambda}, \quad \text { for a given } \epsilon>0 .
$$

Further, on $S_{+}$and $S_{-}$,

$$
|\widetilde{B}(z, z)| \geqq|\operatorname{Re} B(z, z)| \geqq \epsilon(z, z)
$$

so that the lemma of Lax-Milgram (for a statement and proof cf. [69]) can be applied to guarantee the existence of unique $B$-projections $w_{+}^{e}$, w $w_{-}^{e}$ of an arbitrary element $w$ of $H_{2}^{0}$ into the subspace $S_{+}^{\epsilon}$ and $S$ - respectively. If one now forms

$$
w_{0}=w-w_{+}-w_{-}
$$

and defines $S_{0}^{e}$ to be the subspace of all elements $w_{0}$ obtained in this manner, Littman proved the following:

THEOREM. A sufficient condition for the generalized Dirichlet problem to have a solution is that for all $w_{0}^{e}$ in $S_{0}^{e}$,

$$
\left(f_{1}, w_{0}^{0}\right)-B\left(g, w_{0}^{0}\right)=0 .
$$

16 Note the relation to the theory of dissipative operators of $\$ 7$. 
This sufficient condition of Littman's has been obtained as a corollary by Louhivarra [69] from a generalization of a result first proved by Browder. Before stating it, let

$$
E_{k}=\int_{\pi_{k}} d E_{\lambda}, \quad k= \pm 1, \pm 2, \cdots
$$

by the family of projections associated with the above resolutions of the identity for the intervals

$$
\begin{aligned}
& \pi^{k}: \quad \alpha / k \leqq \lambda<\frac{\alpha}{k-1}, \quad k=-1,-2, \cdots, \\
& \alpha /(k+1)<\lambda \leqq \alpha / k, \quad k=+1,+2, \cdots .
\end{aligned}
$$

In terms of this family of projection we have the following:

TheOREM. Let $A=T+i S$ be a closed unbounded transformation, for operators $T$ and $S$ having the same domains with $T$ self-adjoint and $S$ symmetric and let $A^{*}$ be its adjoint. If $E_{k}$ denotes the above family of projections for the operator $\left(A+A^{*}\right) / 2$, then in order for an element $y$ of $\mathrm{H}_{2}$ to be in the range of the transformation $A$ it is sufficient that there exist a sequence of positive integers $k_{j}(j=1,2, \cdots)$ and a sequence $y_{j}{ }^{k}(j=1,2, \cdots)$ for each $k=1,2, \cdots$ of elements from the orthogonal complement of the null space of the transformations so that $\left(y_{j}{ }^{0}=0\right)$

$$
\lim \left(x, y-\sum_{k=-1_{j}}^{+1_{j}} y_{j^{k}}\right)_{2}=0 ; \quad(x, x)_{2}=\|x\|_{2}^{(2)}
$$

for each $x$ in $\mathrm{H}_{2}$ and

$$
\sup _{j>1} \sum_{k=-\infty}^{\infty} k^{2}\left\|y_{j}\right\|_{2}<\infty
$$

In addition there is the following important special case due to Browder [6].

THeOREM. If $A$ is a bounded self-adjoint transformation of the space $\mathrm{H}_{2}$ and $E_{\lambda}$ the corresponding resolution of the identity, then the linear set $M$ of those elements y from $\mathrm{H}_{2}$ for which the integral

$$
\int_{-1}^{1} \frac{d_{\lambda}\left(E_{\lambda} y, y\right)_{2}}{\lambda^{2}}
$$

exists is identical to the range of the transformation $T$.

These results form a mathematical basis for Dirichlet principle for 
scattering problems when they are considered together with the following theorem of Wilcox [112]:

THEOREM. Let $C_{2}$ denote the class of twice continuously differentiable functions satisfying the Helmholtz equation in the closure of a region $V$ exterior to a closed bounded surface regular in the sense of Kellogg and which also satisfy the Sommerfeld radiation condition; then the improper integrals

$$
\int_{V}|u|^{2} d^{8} r
$$

and

$$
\int_{V}|\nabla u|^{2} d^{3} r
$$

are convergent for $\operatorname{Im} k>0$ for all functions $u$ in $C_{2}$.

In particular, in connection with the reformulation of the radiation condition in terms of square integrability as used by Ritt and Kazarinoff [87], the extensions of the above theory to appropriate Hilbert spaces do not appear difficult and it is reasonable to conjecture that this extension of Dirichlet principle to scattering problems will be systematically carried out and evaluated as to its usefulness in the near future.

\section{REFERENCES}

I. N. I. Achieser and I. M. Glasmann, Theorie der linearen Operatoren im Hilbert Raum, Mathematische Lehrbücher, vol. 4, Berlin, Akademie-Verlag, 1954, xiii +369 pp.

II. H. W. Bode, New work analysis and feedback amplifier design, New York, Van Nostrand, 1945, xii +551 pp.

III. N. N. Bogoliubov and D. V. Shirkov, Introduction to the theory of quantized fields, New York, Interscience Publishers, 1959, xvi +720 pp.

IV. E. A. Coddington and N. Levinson, Theory of ordinary differential equations, International Series in Pure and Applied Mathematics, New York, McGraw-Hill, 1955, xii+429 pp.

V. S. Flügge and H. Marshall, Rechenmethoden der Quantentheorie. I. Berlin, Springer, 1952, vii +272 pp.

VI. W. Heitler, The quantum theory of radiation, $3 \mathrm{~d}$ ed., International Series of Monographs in Physics, Oxford, Clarendon Press, 1954, xi+430 pp.

VII. M. J. Lighthill, An introduction to Fourier analysis and generalized functions, Cambridge University Press, 1958, viii +79 pp.

VIII. C. C. Lin, The theory of hydrodynamical stability, Cambridge University Press, 1955.

IX. D. E. Kerr, Propagation of short radio waves, Massachusetts Institute of Technology Radiation Laboratory Series vol. 13, New York, McGraw-Hill, 1951, xvii $+728 \mathrm{pp}$. 
X. W. D. Kupradse, Randwertaufgaben der Schwingungstheorie und Integralgleichungen, Berlin, Akademie Verlag, 1956, viii +239 pp.

XI. P. S. Morse and H. Feshbach, Methods of theoretical physics. I-II, International Series in Pure and Applied Physics, New York, McGraw-Hill, 1953, xxii +997 pp. and xxvii $+979 \mathrm{pp}$.

XII. N. I. Muskhelishvili, Singular integral equations, Groningen, Noordhoff, 1953, vi +447 pp.

XIII. A. Sommerfeld, Partial differential equations in physics, New York, Academic Press, 1949, xi +335 pp.

XIV. M. H. Stone, Linear transformations in Hilbert space and their applications to analysis, Amer. Math. Soc. Colloquium Publications, vol. 15, New York, 1932, $\mathrm{vi}+622 \mathrm{pp}$.

XV. E. C. Titchmarsh, Eigenfunction expansion associated with second-order differential equations, I-II, Oxford, The Clarendon Press, 1946 and 1958, 184 pp. and $\mathrm{vi}+404 \mathrm{pp}$.

XVI. F. G. Tricomi, Integral equations, New York, Interscience Publishers, 1957, viii +238 pp.

1. H. A. Bethe, Nuclear physics, Rev. Mod. Phys. vol. 9 (1937) p. 69.

2. K. Bleuer, Eine neue Method zur Behandlung der longitudenalen und scalaren Photonen, Helv. Phys. Acta vol. 23 (1950) p. 567.

3. D. Bohm and E. P. Gross, Theory of plasma oscillations. A. Origin of mediumlike behavior, Phys. Rev. vol. 75 (1949) p. 1185; B. Excitation and damping of oscillations, Phys. Rev. vol. 75 (1949) p. 1864.

4. H. G. Booker and W. Walkinshaw, The mode theory of tropospheric refraction and its relation to wave guides and diffraction. Meteorological factors in radio wave propagation, The Physical Society, London, 1946, p. 80.

5. H. J. Bremmerman and L. Durand, On analytic continuation and Fourier transformations of Schwartz distributions, Institute for Advanced Study, May, 1959.

6. F. E. Browder, A remark on the Dirichlet problem for non-elliptic self-adjoint partial differential equations, Rend. Circ. Mat. Palermo vol. 6 (1957), p. 249.

7. - On the Dirichlet problem for linear non-elliptic partial differential equations. II, Rend. Circ. Mat. Palermo vol. 7 (1958) p. 303.

8. G. E. Brown, Foundations of the optical model for nuclei and direct interactions, Rev. Mod. Phys. vol. 31 no. 4 (1959) p. 893.

9. T. Carleman, Sur la resolution des certaines équations intégrales, Arkiv. for Mat. Astron. och Fysik. vol. 16 no. 26 (1922) p. 7.

10. K. M. Case, Plasma oscillations, Annals of Physics vol. 7 (1959) p. 349.

11. - Elementary solutions of the transport equation and their applications, Annals of Physics vol. 9 (1960) p. 1.

12. - Stability of inviscid plane couette flow, Phys. Fluids vol. 3 (1960) p. 143.

13. - Stability of an idealized atmosphere, Phys. Fluids vol. 3 (1960) p. 149.

14. - Edge effects and the stability of plane couette flow, Phys. Fluids vol. 3 (1960) p. 432.

15. - Taylor instability of an inverted atmosphere, Phys. Fluids vol. 3 (1960) p. 366.

16. - Lecture on scattering and dispersion theory, 2nd semester, 1959-1960. University of Michigan, Ann Arbor.

17. E. E. Condon and R. W. Gurney, Quantum mechanics and radioactive disintegration, Phys. Rev. vol. 33 (1929) p. 127. 
18. C. L. Dolph, The mathematician grapples with linear problems associated with the radiation condition, I.R.E. Trans. on Antennas and Propagation, AP-4, no. 3 (1956) p. 302.

19. $A$ saddle point characterization of the Schwinger stationary points in exterior scattering problems, J. Soc. Ind. Appl. Math. vol. 5 no. 3 (1957) p. 89.

20. C. L. Dolph, J. McLaughlin and I. Marx, Symmetric linear transformations and complex quadratic forms, Comm. Pure Appl. Math. vol. 7 (1954) p. 621.

21. C. L. Dolph and R. K. Ritt, The Schwinger variational principle for one-dimensional quantum scattering, Math. Z. vol. 65 (1956) pp. 309-326.

22. C. L. Dolph and D. C. Lewis, On the application of infinite systems of ordinary differential equations to perturbations of plane poiseuille flow, Quart. Appl. Math. vol. 26 (1958) p. 97.

23. C. L. Dolph and S. Altshuler, Variational principles for the wave function in scattering theory, Phys. Rev. vol. 109 (1958) p. 1830.

24. C. L. Dolph and F. Penzlin, On the theory of a class of non-self-adjoint operators and its applications to quantum scattering theory, Ann. Acad. Sci. Fennicae no. 263 (1959).

25. W. Feller, The parabolic differential equations and the associated semigroups of transformations, Ann. of Math. vol. 55 (1952) p. 468.

26. B. Friedman and L. I. Mishoe, Eigenfunction expansion associated with a nonself-adjoint differential equation, Pacific J. Math. vol. 6 (1956) p. 249.

27. K. Friedrichs, On the perturbation of continuous spectra, Comm. Pure Appl. Math. vol. 1 (1948) p. 361.

28. G. Gamov, Zur Quanten Theorie des Atomkernes, Z. Physik. vol. 51 (1928) p. 204.

29. - Zur Quanten Theorie der Atomzertrümmerung, Z. Physik vol. 52 (1928) p. 510.

30. P. Garabedian, An integral equation governing electromagnetic waves, Quart. Appl. Math. vol. 16 (1955) p. 428.

31. I. M. Gelfand, On the spectrum of non-self-adjoint differential operators, Uspehi Mat. Nauk vol. 7 no. 6 (52) (1952) p. 183.

32. M. Gell-Mann and M. L. Goldberger, The formal theory of scattering, Phys. Rev. vol. 91 (1953) p. 402.

33. I. M. Glasmann, An analogue of the extension theory of hermitian operators and a non-symmetric one-dimensional boundary-value problem on a half axis, Dokl. Akad. Nauk SSSR vol. 115 (1957) p. 214.

34. - On expandibility according to a system of eigenfunctions of dissipative operators, Uspehi Mat. Nauk vol. 13 no. 3 (81) (1958) p. 179.

35. D. S. Greenstein, On the analytic continuation of functions which map the upper half plane into iself, to appear in J. Math. Analysis and App.

36. S. N. Gupta, Theory of longitudinal photons in quantum electrodynamics, Proc. Phys. Soc. Sect. A vol. 63 (1950) p. 681.

37. D. R. Hartree, J. G. Michel and P. Nicholson, Practical methods for the solution of the equation of trospheric refraction. Meteorological factors in radio wave propagation, Proc. Phys. Soc. Sect. A (1946) p. 127.

38. W. Heisenberg, Die "beobachtbaren Grössen" in der Theorie der Elementarteilchen. I, Z. Physik vol. 120 (1943) p. 513; II, vol. 120 (1943) p. 673.

39. E. Hopf, Über die Anfangswertaufgabe für die hydrodynamischen Grundgleichungen, Math. Nachr. vol. 4 (1952) p. 87. 
40. L. Hulthen, Variational problem for the continuous spectrum of a Schrödinger equation, Kungl. Fysiogr. Sällsk. i Lund Förh. vol. 14 (1944) p. 21.

41. J. Humblet, Sur la definition des niveaux virtuels des noyaux atomiques et l'establissement de la formule de dispersion, Mem. Soc. Roy. Sci. Liège vol. 12 no. 4 (1952).

42. - Definition of the virtual level of the deutron, Phys. Rev. vol. 97 (1955) p. 1145 .

43. J. M. Jauch, Theory of scattering operator, Helv. Phys. Acta. vol. 31 (1958) p. 127.

44. R. Jost and A. Pais, On the scattering of a particle by a static potential, Phys. Rev. vol. 82 (1951) p. 840.

45. T. Kato, Variational methods in collison problems, Phys. Rev. vol. 80 (1950) p. 475 .

46. K. Kodaira, The eigenvalue problem for ordinary differential equations of secondorder and Heisenberg's theory of the s-matrices, Amer. J. Math. vol. 71 (1949) p. 921.

47. N. N. Khuri, Analyticity of the Schrödinger scattering amplitude and nonrelativistic dispersion relations, Phys. Rev. vol. 107 (1957) p. 1148.

48. W. Kohn, Variational methods in nuclear collison problems, Phys. Rev. vol. 74 (1948) p. 1763.

49. - Variational scattering theory in momentum space, Phys. Rev. vol. 84 (1951) p. 495.

50. G. Köthe, Randverteilungen analytischer Funktionen, Math. Z. vol. 57 (1952) p. 13.

51. M. G. Krein, The theory of self-adjoint extensions of semi-bounded Hermitian transformations and its applications, Mat. Sb. (N. S.) vol. 20 (62) (1947) p. 431.

52. - Integral equations on the half-line with a kernel depending on the difference of the arguments, Uspehi Mat. Nauk. vol. 13 no. 5 (83) (1958) p. 3.

53. M. G. Krein and I. S. Iohvidov, Spectral theory in a space of indefinite metric I. Trudy Moskov. Mat. Obšč. vol. 5 (1956) p. 367; Amer. Math. Soc. Translations vol. 13 (1960) p. 105; II, Trudy Moskov. Mat. Obšc. vol. 8 (1959) p. 413.

54. M. G. Krein and I. Gohberg, Fundamental aspects of defect numbers, root numbers and indexes of linear operators, Uspehi Mat. Nauk. (N.S.) vol. 12 no. 2 (74) (1957) p. 43; Amer. Math. Soc. Translations vol. 13 (1960) p. 185.

55. M. G. Krein, Systems of integral equations on the half-line with kernels depending on the difference of the arguments, Uspehi Mat. Nauk. (N.S.) vol. 13 no. 2 (80) (1958) p. 3.

56. W. D. Kupradse, Methode der Integralgleichungen in der Beugungstheorie, Mat. Sb. vol. 41 no. 4 (1934) p. 57.

57. L. Landau, On the vibrations of the electron plasma, J. Phys. vol. 10 no. 1 (1946) p. 25.

58. A. M. Lane, and R. G. Thomas, R-matrix theory of nuclear reactions, Rev. Mod. Phys. vol. 30 (1958) p. 257.

59. Y. W. Lee, Synthesis of electric networks by means of the Fourier transform of Laguerre functions, J. Math. Phys. vol. 11 (1932) p. 83.

60. J. Lehner and G. M. Wing, On the spectrum of an unsymmetric operator arising in the transport theory of neutrons, Comm. Pure. Appl. Math. vol. 8 (1955) p. 217.

61. - Solution of linearized Boltzmann transport equation for slab geometry, Duke Math. J. vol. 23 (1956) p. 125.

62. H. Levine, Variational principles for solving electro-magnetic boundary value problems, Notes of lectures, 1954, Sylvania Products Co., Mountain View, California. 
63. V. B. LidskiY, On the completeness of the system of eigen and associated functions of a non-self-adjoint differential operator, Dokl. Akad. Nauk SSSR (N.S.) vol. 110 (1956) p. 172.

64. - Conditions for the completeness of systems of root spaces having a nonself-adjoint operator with discrete spectra, Trudy. Moskov. Obšx. vol. 8 (1959) p. 83.

65. B. A. Lippman and J. Schwinger, Variational principles for scattering operators, Phys. Rev. vol. 79 (1950) p. 469.

66. W. Littman, Remarks on the Dirichlet problem for general linear partial differential equations, Comm. Pure Appl. Math. vol. 11 (1958) p. 145.

67. M. S. Livsíc, On spectral decomposition of linear non-self-adjoint operators, Translations Amer. Math. Soc. II vol. 5 (1957) p. 67.

68. I. S. Louhivarra, Über das Dirichletsche Problem für die selbstadjungierten linearen partiellen Differentialgleichungen zweiter Ordnung, Rend. Circ. Mat. Palermo vol. 5 (1956) p. 260.

69. - Ü̈ber verschiedene Metriken in linearen Räumen, Ann. Acad. Sci. Fennicae no. 282 (1960).

70. S. T. Ma, Interpretation of the virtual level of the deutron, Rev. Mod. Phys. vol. 25 (1953) p. 853.

71. G. MacFarlane, Variational methods for determining eigenvalues of the wave equation of anomalous propagation, Proc. Cambridge Philos. Soc. vol. 43 (1947) p. 213.

72. S. Mandelstam, Determination of the pion-nucleon scattering amplitude from dispersion relations and unitarity, Phys. Rev. vol. 112 (1958) p. 1344.

73. N. Marcuvitz, Field representations in spherically stratified regions, Comm. Pure Appl. Math. vol. 4 (1951) p. 263.

74. $\mathrm{H}$. Moses, The scattering operator and the adiabatic theorem, Nuovo Cimento vol. 10 No. 1 (1955) p. 103.

75. B. R. Mukminov, On expansion with respect to eigenfunctions of dissipative kernels, Dokl. Akad. Nauk. SSSR (N.S.) vol. 99 (1954) p. 499.

76. C. Müller, Zur Methode der Strahlungskapazität von H. Weyl, Math. Z. vol. 56 (1952) p. 80.

77. M. Naimark, Investigation of the spectra and expansion theorem of non-selfadjoint differential operators on a semi-axis, Trudy. Moscov. Obšc. vol. 3 (1954) p. 181.

78. - Spectral analysis of non-self-adjoint operators, Uspehi Mat. Nauk vol. 11 no. 6 (72) (1956) p. 183.

79. R. Nevanlinna, Über metrische lineare Räume, Ann. Acad. Sci. Fennicae. I, no. 108 (1952); II, no. 113 (1952); III, no. 115 (1952); IV, no. 163 (1954); V, no. 222 (1956).

80. R. Peierls, Expansions in terms of sets of functions with complex eigenfunctions, Proc. Cambridge Philos. Soc. vol. 44 (1948) p. 242.

81. - Complex eigenvalues in scattering theory, Proc. Roy. Soc. London Sect. A vol. 253 (1959) p. 16.

82. R. Peierls and P. L. Kapur, The dispersion formula for nuclear reactions, Proc. Roy. Soc. London Sect. A vol. 166 (1938) p. 277.

83. R. S. Phillips, Linear differential operators of second order, Research Report EM-42, New York University, 1952.

84. - Dissipative operators and hyperbolic systems of partial differential equations, Trans. Amer. Math. Soc. vol. 90 (1959) p. 193.

85. J. Plemelj, Ein Ergänzungsatz zur Cauchyschen Integraldarstellung analytischer Funktionen, Randwerte betreffend, Monatsh. Math. Phys. vol. 19 (1908) p. 205. 
86. L. K. Prandit, Linear vector spaces with indefinite metric, Nuovo Cimento Supp. XI ser. X (1959) p. 157.

87. R. K. Ritt and N. D. Kazarinoff, On the theory of scalar diffraction and its application to the prolate spheroid, Ann. Physik. vol. 6 (1959) p. 277.

88. - Scalar diffraction theory and the turning point problem, Arch. Rat. Mech. Anal. vol. 5 (1960) p. 177.

89. I. Schensted, Expansions theorems associated with the Orr-Sommerffeld equation and hydrodynamic stability, Thesis, University of Michigan, 1960.

90. J. Schwartz, Perturbations of spectral operators and applications. I. Bounded perturbations, Pacific J. Math. vol. 4 (1952) p. 415.

91. A. J. F. Siegert, On the derivation of the dispersion formula for nuclear reactors, Phys. Rev. vol. 56 (1939) p. 750.

92. A. R. Sims, Secondary conditions for linear differential operators of the second order, J. Math. Mech. vol. 6 (1957) p. 247.

93. J. A. Sparenberg, Applications of the theory of sectionally holomorphic functions to Wiener-Hopf type integral equations, Proc. Akad. Van. Wet. Amsterdam, A, vol. 59 (1956) p. 29.

94. - Applications of the Hilbert problem to problems of mathematical physics, Dissertation, Tech. Hochschule Delft, 1958.

95. H. G. Tillmann, Randverteilungen analytischer Funktionen und Distributionen, Math. Z. vol. 59 (1953) p. 61.

96. E. C. Titchmarsh, Some theorems on perturbation theory. III, Proc. Roy. Soc. London Sect. A vol. 207 (1951) p. 321.

97. —- Eigenfunction expansions associated with partial differential equations, Proc. London. Math. Soc. vol. 5 (1958) p. 1.

98. N. G. Van Kampen, On the theory of stationary waves in a plasma, Physics vol. 21 (1955) p. 949.

99. —- The dispersion equation for plasma waves, Physics vol. 23 (1957) p. 641.

100. V. Weisskopf, H. Feshbach and C. E. Porter, Model for nuclear reactions with neutrons, Phys. Rev. vol. 96 (1954) p. 448.

101. H. Weyl, Über gewöhnliche Differentialgleichungen mit Singularitäten und die zugehörigen Entwicklungen willk ülicher Funktionen, Math. Ann. vol. 68 (1910) p. 220.

102. - Kapazität von Strahlungsfeldern, Math. Z. vol. 55 (1952) p. 187.

103. H. Widom, Equations of the Wiener-Hopf type, Illinois J. Math. vol. 2 (1958) p. 261.

104. A. S. Wightman, Quantum field theory in terms of vacuum expectation values, Phys. Rev. vol. 101 (1956) p. 860.

105. A. S. Wightman and D. Hall, $A$ theorem on invariant analytic functions with applications to relativistic quantum field theory, K. Danske, Nied. Mat. Fy. Med. A. S. vol. 231 (1957) p. 31.

106. E. P. Wigner, On the class of analytic functions from the quantum theory of collisions, Ann. of Math. vol. 53 (1951) p. 36.

107. - On the development of the compound nucleus model, Amer. J. Phys. vol. 23 (1955) p. 371.

108. E. P. Wigner and V. Weisskopf, Über die naturliche Linenbreite in der Strahlung der harmonischen Oszillators, Z. Physik. vol. 65 (1930) p. 18.

109. - Berechnung der natürlichen Linenbreite auf Grund der Diracschen Lichttheorie, Z. Physik. vol. 63 (1930) p. 54.

110. E. P. Wigner and L. Eisenbud, Higher angular momenta and long range interaction in resonance reactions, Phys. Rev. vol. 72 (1947) p. 29. 
111. E. P. Wigner and J. von Neumann, Significance of Loewner's theorem in quantum theory of collisions, Ann. of Math. vol. 59 (1954) p. 418.

112. C. H. Wilcox, $A$ generalization of theorems of Rellich and Atkinson, Proc. Amer. Math. Soc. vol. 7 (1956) p. 27.

113. - Spherical means and radiation conditions, Arch. Rat. Mech. Anal. vol. 3 (1959) pp. 133-148.

114. C. H. Wilcox and P. Hartman, On the solutions of the Helmholtz equation in exterior domains, MRC Tech. Summary Report 149, University of Wisconsin, 1960, pp. 1-37.

115. T. Ikebe, Eigenfunction expansions associated with the Schrödinger operators and their applications to scattering theory, Arch. Rat. Mech. Anal. vol. 5 no. 1 (1960) p. 1.

116. A. Povzner, On the expansions of arbitrary functions in terms of the operator $-\nabla^{2} u+c u$ (Russian), Mat. Sb. vol. 32 (74) (1953) p. 109.

117. T. Kato, Fundamental properties of Hamiltonian operators of Schrödinger type, Trans. Amer. Math. Soc. vol. 70 (1951) p. 195.

118. S. Kuroda, On the existence and the unitary properties of the scattering operator, Nuovo Cimento vol. 12 (1959) p. 431.

UNIVERSITY OF Michigan 